Manuscript 3 of 3

\title{
Development and applications of sialoglycan-recognizing probes (SGRPs) with defined specificities: exploring the dynamic mammalian sialoglycome
}

Saurabh Srivastava ${ }^{1,2}$, Andrea Verhagen ${ }^{1,2}$, Aniruddha Sasmal ${ }^{1,2}$ Brian R. Wasik ${ }^{3}$, Sandra Diaz ${ }^{1,2}$, Hai Yu ${ }^{4}$, Barbara A. Bensing ${ }^{5}$, Naazneen Khan ${ }^{1,2}$, Zahra Khedri ${ }^{1,2}$, Patrick Secrest ${ }^{1,2}$, Paul Sullam $^{5}$, Nissi Varki ${ }^{1,2}$, Xi Chen ${ }^{4}$, Colin R. Parrish ${ }^{3}$ and Ajit Varki ${ }^{1,2 *}$

${ }^{1}$ Department of Cellular and Molecular Medicine, School of Medicine, and ${ }^{2}$ Glycobiology Research and Training Center, University of California, San Diego, San Diego, CA, ${ }^{3}$ College of Veterinary Medicine, Cornell University, Ithaca, NY, ${ }^{4}$ Department of Chemistry, University of California, Davis, Davis, CA, ${ }^{5}$ Department of Medicine, University of California, San Francisco and VA Medical Center, San Francisco, CA

Running Title: Sialoglycan-Recognizing Probes (SGRPs)

*Address correspondence to:

Ajit Varki

UCSD School of Medicine, La Jolla, CA 92093-0687

Phone: (858) 534-2214

Fax: (858) 534-561 
bioRxiv preprint doi: https://doi.org/10.1101/2021.05.28.446202; this version posted May 28, 2021. The copyright holder for this preprint (which

was not certified by peer review) is the author/funder, who has granted bioRxiv a license to display the preprint in perpetuity. It is made available under aCC-BY-NC-ND 4.0 International license.

\section{Keywords}

Sialic acid, Lectin, Adhesin, Bacterial toxin, Acetylation, Sialoglycan-Recognizing Probes,

Sialoglycans, Hemagglutinin-Esterase, $\mathrm{O}$-Acetylation, $\mathrm{N}$-Glycolylneuraminic acid; $\mathrm{N}$ -

Acetylneuraminic acid 


\begin{abstract}
(250words)
Glycans that are abundantly displayed on vertebrate cell surface and secreted molecules are often capped with terminal sialic acids (Sias). These diverse 9-carbon-backbone monosaccharides are involved in numerous intrinsic biological processes. They also interact with commensals and pathogens, while undergoing dynamic changes in time and space, often influenced by environmental conditions. However, most of this sialoglycan complexity and variation remains poorly characterized by conventional techniques, which often tend to destroy or overlook crucial aspects of Sia diversity and/or fail to elucidate native structures in biological systems i.e., in the intact sialome. To date, in situ detection and analysis of sialoglycans has largely relied on the use of plant lectins, sialidases or antibodies, whose preferences (with certain exceptions) are limited and/or uncertain. We took advantage of naturally-evolved microbial molecules (bacterial adhesins, toxin subunits and viral hemagglutinin-esterases) that recognize sialoglycans with defined specificity to delineate 9 classes of Sialoglycan Recognizing Probes (SGRPs: SGRP1-SGRP9) that can be used to explore mammalian sialome changes in a simple and systematic manner, using techniques common in most laboratories. SGRP candidates with specificity defined by sialoglycan microarray studies were engineered as tagged probes, each with a corresponding non-binding mutant probe as a simple and reliable negative control. The optimized panel of SGRPs can be used in methods commonly available in most bioscience labs, such as ELISA, Western Blot, flow cytometry and histochemistry. To demonstrate the utility of this approach, we provide examples of sialoglycome differences in tissues from C57BL/6 wild type mice and human-like $\mathrm{Cmah}^{-/-}$mice.
\end{abstract}




\section{Introduction}

All cells in nature are covered with a dense and complex array of sugar chains (1), and in vertebrates the outermost ends of the branches on this glycan forest are often capped with monosaccharides called sialic acids (Sias), which have enormous intrinsic complexity (2, 3). Most current methods to study this important and dynamic aspect of the glycome are too specialized for an average scientist to employ, and many aspects of this class of important molecules are thus poorly studied, even by experts. Given their ubiquitous presence and terminal position, Sias have been exploited as primary, transient or co-receptors by a diverse range of commensal or pathogenic microorganisms $(4,5)$. These interactions are typically mediated by microbial proteins that have evolved with high binding specificity towards sialoglycans and can differentiate their target Sias by types, modifications, substitutions and/or linkage to underlying glycans.

In this report, we undertake a strategy to develop Sia-specific probes from such microbial proteins that have naturally evolved to recognize Sia complexity with great specificity, likely because of the ongoing evolutionary arms race between microbes and hosts. We have harnessed the Sia specificity of microbial proteins, and if found insufficient assessed other available probes to generate a simple and reliable toolkit that can be used to easily monitor dynamic changes of the sialic acids in normal and abnormal states. Specifically, this study advances a set of comprehensive sialoglycan-recognizing probes (SGRPs) that can confirm whether a biological sample has any sialic acids or not; if so, the type of common Sia variations (particularly O-acetylation), the linkage to underlying glycans, and the presence of $N$-acetyl or $N$-glycolyl groups. To identify candidates for such an updated set of probes for mammalian Sias, we assessed a number of Sia-specific binding proteins, novel or reported in accompanying or previous articles for their specificities towards different classes of mammalian Sia types and/or linkage to the underlying glycans. 
Specifically, 9 types of SGRPs were defined from a subset of proteins including bacterial serinerich repeat (SRR) adhesins, bacterial B5 toxins, viral hemagglutinins (HAs) and hemagglutininesterases (HEs) and compared them to previously known invertebrate and plant lectins, selected Siglecs, and polyclonal and monoclonal antibodies (MAbs). Upon identification of the best SGRP for each class of sialoglycans, a mutant inactive probe was also developed as an internal control of each probe's specificity. To ensure minimal loss of sensitive Sia modifications/substitutions, experimental conditions were optimized, and the specificity of each probe was tested with positive and negative controls, such as pretreatment with specific sialidases or esterases, or mild periodate oxidation of the Sia side chain. The binding specificities of SGRPs were confirmed by testing on a sialoglycan microarray, constituting a diverse array of more than 100 mammalian sialoglycans and demonstrated by examples of generalized laboratory methods of ELISA, Western blotting, and fluorescence detection by flow cytometry and histological analysis. An example of application of SGRPs is provided, showing sialoglycome changes in mice with human-like loss of the CMAH enzyme.

\section{Results and Discussion}

Defining distinct classes of Sialoglycan Recognizing Probes (SGRPs). We sought to define a set of SGRPs for detection of the most common types of mammalian sialoglycan variants, along with non-binding mutants as controls. This approach simplifies in-situ detection for all major types of Sias (SGRP1), typical $N$-acyl modifications at C-5 of Sias (SGRP2, SGRP5), linkages to underlying glycans (SGRP3, SGRP6, SGRP8) and occurrence of $O$-acetyl groups (SGRP4, SGRP7, SGRP9). We also suggested a SGRP numbering system (see Table 1) for these probes that make them easier to remember. To define a given probe, we used a superscript, and added NB 
for the non-binding control. Thus, for example, the Y. enterocolitica toxin B subunit (YenB) that recognizes all Neu5Ac and Neu5Gc forms and linkages is designated SGRP1 ${ }^{\text {YenB }}$ and the nonbinding variant as SGRP1 ${ }^{Y e n B} \mathrm{NB}$. The current set of SGRPs does not include probes specific for 2-keto-3-deoxy-D-glycero-D-galacto-nononic acid $(\mathrm{Kdn})$, which, although naturally found in mammals, occurs in limited amounts and primarily in the free form (6).

Search for naturally evolved microbial molecules with defined specificity towards specific aspects of sialoglycans. The SGRP candidates were defined by sialoglycan microarray studies, with each probe accompanied by a corresponding non-binding mutant as a negative control. The essential criterion for a protein to qualify as an SGRP was that it must show specificity towards the preferred Sia modification/linkages. The detailed analyses of most updated contemporary set of probes (SGRPs) and specificities and their practicability towards probing mammalian sialoglycans is discussed in subsequent sections. As mentioned in Table 1, we could not identify any microbial candidate having a better or broader specificity for SGRP5 ( $N$-glycolyl-Sias) than our previously described affinity--purified Neu5Gc chicken polyclonal IgY, or SGRP6 Sambucus nigra agglutinin (SNA), the conventionally used lectin probe for $\alpha 2-6$-linked Sias.

SGRP1 $^{\text {YenB }}$ is a probe to detect all mammalian sialic acids. While $\alpha 2-3$ Sia-binding MAL and a2-6Sia-binding SNA together recognize the majority of Sia linkages, their preferences cannot be generalized for all types of Sias. Previously, WGA and LFA have been reported as having broadspectrum Sia specificity, but their preferences towards Neu5Ac bring into question their utility as practicable probes to detect all types of Sias (7-9). For an SGRP that binds all types of Sia, we first considered YpeB (Yersinia pestis Toxin B subunit that recognizes both Neu5Ac- and Neu5Gc-terminated glycans-detailed sialoglycan preferences are reported in the companion paper 1 (Khan et al., see also Figure S1). However, despite its ability to recognize most major 
classes of mammalian sialoglycans in glycan array and serum ELISAs, YpeB did not bind 4-OAcSias (Suppl. Fig S1). Relying on the promising lead from YpeB, we investigated additional B subunits of AB5 bacterial toxins and selected YenB (Y. enterocolitica toxin B subunit) based on homology with YpeB and S. typhimurium ArtB, and broad host specificity (see companion paper, Sasmal et.al.). Using His6-tagged YenB (see Sasmal et al. accompanying paper) we confirmed the protein's recognition for both Neu5Ac and Neu5Gc including 9-O- and 4-O-acetylated Sias, a clear advantage over WGA, LFA and our initial candidate YpeB (Suppl. Figure S2). Notably, YenB did not show any binding to non-sialylated glycans in the same assay, further confirming its extensive but Sia-selective binding to glycans. In order to biotinylate YenB without affecting its Sia-binding domain, we attempted to clone YenB with additional tags for biotinylation (SNAP, ACP and Avi) but the modifications resulted in poor protein quality and yield, leading to reduced binding of Sia in glycan arrays. A direct $N$-hydroxysuccinimide (NHS-)-biotin conjugation of YenB was therefore optimized to obtain the final biotinylated probe (SGRP1 ${ }^{\text {YenB }}$ ) that showed no loss of binding preferences in comparison to non-biotinylated YenB. It was previously established that a serine residue contributes critically to Neu5Ac binding, while a tyrosine residue interacts with the extra $\mathrm{OH}$ group at the $\mathrm{C} 5$-acyl chain of Neu5Gc and is thus critical for its binding (10). As described in a companion paper (Sasmal et.al.), we aligned the YenB sequence with those of $E$. coli SubB and S. typhimurium ArtB and predicted the conserved serine (S31) and tyrosine (Y100) in YenB. Mutating these critical sites for Sia recognition (YenB, S31A;Y100F) produced SGRP $1^{\text {YenB }}$ NB as an internal control for Sia-binding SGRP1 ${ }^{\text {YenB }}$ (Suppl. Fig. S3A \&B). The final binding and nonbinding specificities for SGRP1 ${ }^{\text {YenB }}$ were tested on a sialoglycan microarray with nearly 130 mammalian sialoglycan types, suggesting all-inclusive Sia specificity in SGRP1, and a complete lack of Sia-binding by SGRP1 ${ }^{\operatorname{Yen} B} \mathrm{NB}$ (Suppl. Fig S4). Since there were no other 
molecules known to possess YenB-like Sia specificities, the pair of SGRP1 ${ }^{Y e n B}$ and SGRP1 ${ }^{\text {YenB }}{ }^{\mathrm{NB}}$ are currently the most appropriate probes to detect all mammalian Sia types. The utility of SGRP $1^{\text {YenB }}$ as a useful tool of in situ Sia detection through ELISAs, Western Blotting, IHC and flow cytometry is described and discussed below.

SGRP2 ${ }^{P l t B}$ recognizes all Neu5Ac-terminated glycans. Neu5Ac is the most abundant Sia type and occurs in all Sia-expressing organisms. Mammals (including humans) that lack a functional CMAH enzyme have lost the ability to convert cytidine 5'-monophosphate $N$-acetylneuraminic acid (CMP-Neu5Ac) into CMP-Neu5Gc, and express excess Neu5Ac as their primary Sia type. Despite such wide distribution and roles in human physiology/immunity, there has so far been no direct probe to selectively detect all forms and linkages of Neu5Ac in situ. Among commonly known probes for sialoglycans, WGA shows relative specificity towards Neu5Ac-glycans but neither recognizes all sialoglycans terminating with Neu5Ac, nor is it exclusive to Neu5Ac, showing a dual preference for Neu5Ac and GlcNAc (8) (Suppl Fig. S2A). Previously, we identified Neu5Ac-specific binding in PltB, the B subunit of Typhoid toxin which preferred to bind human erythrocytes and tissues rich in Neu5Ac over the corresponding Neu5Gc-rich samples from Chimpanzees (11). Similar Neu5Ac-specific patterns of PltB binding were further studied recently by others who also reported its binding $O$-acetylated Neu5Ac in both $\alpha 2-3$ and $\alpha 2-6$-linked sialoglycans (12). In the accompanying paper (Sasmal et al.), we report on the binding of PltB to a range of naturally occurring Neu5Ac but not Neu5Gc-glycans, underlining PltB's appropriateness as a Neu5Ac-recognizing probe. We cloned the His 6 -tagged Neu5Ac-binding domain of PltB with an additional ACP tag (NEB) for biotinylation, but we then had to abandon this approach due to poor expression and purification quality of the resulting protein. The final probe version of PltB (SGRP2 ${ }^{P l t B}$ ) was derived by direct biotinylation of PltB with NHS-biotin. 
This biotinylated-PltB shows comparable specificity and avidity towards Neu5Ac glycans as the unmodified protein (Fig 1). The Neu5Ac-binding domain of PltB was characterized previously and a serine residue was reported to be critical for Neu5Ac-binding by PltB (11). Thus PltB ${ }^{\mathrm{S} 35 \mathrm{~A}} \mathrm{NB}$, an internal nonbinding mutant control of SGRP2 was also produced, biotinylated and included in all studies (Suppl. Fig S3 C \& D).

The sialoglycan microarray with mammalian sialoglycan types showed the Neu5Acspecific binding pattern of SGRP2 $2^{P l t B}$ while SGRP2 $2^{P l t B}$ NB completely lacks binding (Suppl. Fig S4). In agreement with previous data $\operatorname{SGRP}^{P l t B}(11)$, high binding preferences towards Neu5Ac were seen regardless of substitutions, modifications, or linkages to underlying glycans. As shown in the array, SGRP2 $2^{P l t B}$ demonstrates comparable binding for $\alpha 2-3, \alpha 2-6$ and $\alpha 2-8$ linked Neu5Ac and clearly distinguishes them from $\alpha 2-3, \alpha 2-6$ or $\alpha 2-8$ linked Neu5Gc (Fig 1). Notably, $\mathrm{SGRP}^{P l t B}$ also exhibited recognition for $\mathrm{Neu} 4,5 \mathrm{Ac}_{2}$ and $\mathrm{Neu} 5,9 \mathrm{Ac}_{2}$. Taken together, SGRP2 $2^{P l t B}$ showed definite superiority over WGA as Neu5Ac binding probe (Suppl Fig S2A). We therefore prepared SGRP2 $2^{P l t B}$ and a SGRP2 $2^{P l t B} \mathrm{NB}$ control as Neu5Ac-binding and non-binding probes for use in general methods such as ELISAs, Western blotting, flow cytometry and IHC.

SGRP3 $^{\text {Hsa }}$ selectively recognizes $\boldsymbol{\alpha 2 - 3 - l i n k e d ~ S i a s . ~ L e c t i n s ~ f r o m ~ M a a c k i a ~ a m u r e n s i s ~ s e e d s , ~ f i r s t ~}$ reported by Kawaguchi et al (13) have been the gold standard for detection for Sias $\alpha 2-3$-linked to penultimate Gal. These lectins initially designated as 'strongly mitogenic M. amurensis leukoagglutinin (MAL) and 'strongly hemagglutinating M. amurensis hemagglutinin' (MAH) are commonly known as MAL-I and MAL-II, respectively. While both lectins require at least a Sia 2 3Gal disaccharide structure to bind, they differ in their requirement for specific structures. MALI shows stronger binding to Sia $\alpha 2-3 \mathrm{Gal} \beta 1-4 \mathrm{GlcNAc} / \mathrm{Glc}$ (a trisaccharide common in N-glycans) and MAH (MAL-II) is selective towards Sia 2 2-3Galß1-3GalNAc, typically found in O-linked 
glycans. Among Siaa2-3-linked structures tested, MAH prefers 9- $O$-acetyl Sias with Neu5Ac over Neu5Gc and Ser/Thr-linked O-glycan structures (14-16). However, MAL-I can also recognize 3$O$-sulfated Gal terminated oligosaccharides i.e., it does not show exclusivity towards sialylated sequences (17). Instead, MAL-I displays widespread affinity towards $\alpha 2-3$-linked Neu5Ac and Neu5Gc, but with selective inclination towards Asn-linked over Ser/Thr-linked glycans. Attempts had been made to improve Maackia lectins (18) but this did not yield a general-purpose Sia 2 -3recognizing probe.

We looked into the Sia-binding properties of previously reported Siglec-like-domaincontaining ligand-binding regions (BRs) of Streptococcus gordonii SRR adhesins (19-21). In particular, we selected GspB-BR of $S$. gordonii strain M99, Hsa-BR of S. gordonii Stain DL1 (Challis), and UB10712-BR of S. gordonii strain UB10712, and investigated their suitability for a comprehensive Siaa2-3-linkage identifying probe. All 3 BRs were expressed as SNAPf-His 6 fusions in the pGEX-3X vector in a bacterial expression system along with their non-binding variants HsaBR (R340E), GspBBR (R484E) and UB10712BR (R338E) (Suppl Fig S3).

The pairs of $\mathrm{Hsa}_{\mathrm{BR}} / \mathrm{Hsa}_{\mathrm{BR}} \mathrm{NB}, \mathrm{GspB}_{\mathrm{BR}} / \mathrm{GspB}_{\mathrm{BR}} \mathrm{NB}$ and UB10712 ${ }_{\mathrm{BR}} / \mathrm{UB} 10712_{\mathrm{BR}} \mathrm{NB}$ were biotinylated using SNAP-benzyl guanine chemistry and tested for their sialoglycan binding on our microarray. Despite their structural similarities, the 3 biotinylated BRs displayed uniquely different ligand binding profiles, including differential recognition of sialyl Lewis antigens and sulfated glycans. While GspB BR selectively binds sialyl-T antigen (Neu5Ac $\alpha 2-3 \mathrm{Gal} \beta 1-3 \mathrm{GalNAc})$ and related structures, Hsabr displays broader specificity covering NeuAc $\alpha 2-3 \mathrm{Gal} \beta 1-4 \mathrm{GlcNAc}$ and sialyl-T antigen (Fig 1 \& Suppl Fig S5A) (20-22). In comparison to HsaBR, GspB BR $_{\text {imparts }}$ lesser specificity towards $\alpha 2-3$ than $\alpha 2-6$ Sia linkages, (20) and falls short of the binding range of $\mathrm{Hsa}_{\mathrm{BR}}$, which not only includes trisaccharide and oligosaccharide but also disaccharide Sias. 9-O- 
Acetylation on Sia did not block $\mathrm{GspB}_{\mathrm{BR}}$ or Hsa $\mathrm{BR}_{\mathrm{BR}}$ binding, but sulfation appeared to enhance Hsa $_{\mathrm{BR}}$ binding (21). Sia binding preferences of UB10712 ${ }_{\mathrm{BR}}$ remained comparable to $\alpha 2-3$ linked Sia specificities of $\mathrm{Hsa}_{\mathrm{BR}}$ and $\mathrm{GspB}_{\mathrm{BR}}$. UB10712 $\mathrm{BR}$ bound to range of $\alpha 2-3$ Sia linkages including sialyl Lewis X, 3'-sialyllactosamine and their sulfated forms but preferred Neu5Ac over Neu5Gc sequences generally (Suppl Fig S5A) (19)). Confirming the Sia-specificity of these BRs, the NB variants did not show any binding of Sia linkages/modifications on the array (Suppl Fig S4, S5). The data obtained with biotinylated BRs also agrees with previously published reports on GSTfusion BRs (19-22), and the comparable binding abilities of these biotinylated probes with the original BRs confirmed that biotinylation did not affect the Sia specificity of these proteins.

Considering the results with biotinylated-HsaBR on the sialoglycan microarray, we asked if Hsa $_{\mathrm{BR}}$ could be a replacement for MAL I and MAH, conventional lectins for this class of probes (SGRP3). In an experiment to compare Siao2-3-binding preferences of Biotin-Hsabr, Biotin-MAL and Biotin-MAH, Hsa Sia modifications ( $O$-acetylation, $O$-sulfation), or glycan structures (disaccharides, trisaccharides, oligosaccharides) (Suppl Fig S6A). Taken together with the glycan array data, we chose biotinylated $\mathrm{Hsa}_{\mathrm{BR}}$ as our SGRP 3 probe $\left(\mathrm{SGRP}^{H s a}\right)$ and and its non-binding variant Hsa ${ }^{\mathrm{BR}}$ (R340E) as the nonbinding control (SGRP3 $\left.{ }^{H s a} \mathrm{NB}\right)$ to characterize $\alpha 2-3$ Sia-binding through various assays such as ELISA, Western Blotting, FACS and histochemistry, discussed below.

SGRP4 $^{M H V}$ as a probe for 4-OAc-Sias. A major contributor to mammalian sialoglycan diversity is $O$-acetylation, substituting the sialic acid hydroxyl groups at $\mathrm{C} 4, \mathrm{C} 7, \mathrm{C} 8$, and/or $\mathrm{C} 9(23,24)$. The presence or absence of these $O$-acetyl moieties can block or promote binding of cellular and microbial lectins, and their regulation through sialate-O-acetylesterases (SOAEs) and sialate-Otransferases (SOATs) act as the molecular switches to control several cellular functions and 
interactions $(25,26)$. In contrast to $O$-acetylation at $\mathrm{C} 7$ or $\mathrm{C} 9-\mathrm{OH}$, the occurrence of $4-O$-acetylated Sia and its structural and functional significance have yet to be explored in much detail. In general, 4-OAc-Sias have been difficult to study even by chemical methods, due to variable expression or absence in many animal species, dynamic occurrence in pathological conditions, resistance to conventional sialidases, lability to acidic conditions (27) and masking of Sias from detection by some lectins. Equine erythrocytes, $\alpha 2$-macroglobulins and sera were used to study 4-OAc-“HD3”reactive antibodies due to their high 4-O-acetyl- $\mathrm{N}$-acetylneuraminic acid $\left(\mathrm{Neu} 4,5 \mathrm{Ac}_{2}\right)$ content (30$50 \%$ of total Sias) (28). Guinea pigs serve as another common source of mammalian 4-OAc-Sias. $\mathrm{Neu} 4,5 \mathrm{Ac}_{2}$ comprises a considerable share in serum (30\% of all Sias) and liver ( $10 \%$ of all Sias) Sias in guinea pig, besides traces of 4-O-acetyl- $\mathrm{N}$-glycolylneuraminic acid (Neu5Gc4Ac) in serum (29).

In humans, 4-OAc has been reported in tumor-associated antigens of colon cancers, melanomas and gastric cancers using "HD" antigen specific antibodies, for example a chicken antibody specific to Neu5Gc4Ac-lactosylceramide (4-OAc-HD3) recognized Neu5Gc4Ac in GM3 ganglioside fractions of human colon cancer tissues (30). Similar 4-OAc-HD3-reactive HD antibodies have also been reported in sera of patients suffering from malignancies and liver diseases (31). Two chicken MAbs HU/Ch2-7 and HU/Ch6-1 reacted with Neu5Gc4Ac in HD3 antigens (32). These MAbs, specific for individual glycan structure were a major tool for detection of 4-OAc in gangliosides or glycoproteins. Despite frequent reports on heterogenous 4-OAc in HD antigens in human cancers, there has not been a reliable conventional probe for in situ detection of this entire class of sialoglycans.

Previously, a sialic acid-binding lectin with specificity for $O$-acetyl Sia was purified from the hemolymph of the California coastal crab Cancer antennarius, which was more precise than 
other known lectins from horseshoe crab (Limulus Polyphemus) and slug (Limax flavus) but it also showed affinity towards 9- $O$-acetyl in addition to 4-O-acetyl Sias (33). A lectin from Tritrichomonas foetus, a parasitic protozoan that causes abortion in cows, was reported to react preferentially with $\mathrm{Neu} 4,5 \mathrm{Ac}_{2}$ over de-O-acetylated Sias, and agglutinated equine erythrocytes containing $\mathrm{Neu} 4,5 \mathrm{Ac}_{2}$ efficiently, but its preferences were also not exclusive for 4-O acetylated Sias (34).

In general, $O$-acetylation of Sias can be a major receptor determinant for some viruses. Among the first viruses shown to initiate infections via O-Ac-Sias were the influenza $\mathrm{C}$ viruses, human coronavirus OC43, Bovine Coronaviruses $\mathrm{BCoV}$ and porcine encephalomyelitis virus (PToV), but none of them exhibited binding of Neu4,5Ac (35-39). Infectious salmon anemia virus (ISAV), the causative agent of infections in Atlantic salmons showed specificity for, and hydrolysis of 4-OAcetylatedAc-Sias (40). ISAV preferentially de-O-acetylated free and glycosidically bound $\mathrm{Neu} 4,5 \mathrm{Ac}_{2}$ and showed lower and no hydrolysis for free and bound $\mathrm{Neu} 5,9 \mathrm{Ac}_{2}$ respectively. ISAV exhibited hydrolysis for both $\mathrm{Neu} 4,5 \mathrm{Ac}_{2}$ and $\mathrm{Neu} 4 \mathrm{Ac} 5 \mathrm{Gc}$ at comparable efficacy, which was a significant advantage over known 4-OAc-Sia-binding molecules but it's affinity for free $\mathrm{Neu} 5,9 \mathrm{Ac}_{2}$ although lower than influenza C virus (40), restricted possibilities to derive a comprehensive probe for 4-OOAc-Sias.

Murine coronavirus mouse hepatitis virus (MHV-stain S) expresses a hemagglutininesterase that exhibits comparable sialate-4-O-Acetylesterase enzymatic activity to that of ISAV (41). Unlike comparable esterases from other sources, MHV-S HE protein specifically de-Oacetylates $\mathrm{Neu} 4,5 \mathrm{Ac}_{2}$ but not $\mathrm{Neu} 5,9 \mathrm{Ac}_{2}$, and converts glycosidically-bound $\mathrm{Neu} 4,5 \mathrm{Ac}_{2}$-rich glycoproteins from horse and guinea pigs to Neu5Ac (41). MHV-S was able to hydrolyze acetyl esters from free as well as glycosidically-linked $\mathrm{Neu} 4,5 \mathrm{Ac}_{2}$. Interestingly, MHV-A59 and several 
other MHV strains do not express a HE (42-44). Previously, the MHV-S HE ectodomain, released from HE-Fc by thrombin- cleavage, was reported to exhibit proper sialate-4-O-acetylesterase activity when assayed for substrate specificity with a synthetic di- $O$-acetylated Sia (4,9-di-Oacetyl- $N$-acetylneuraminic acid $\alpha$-methylglycoside, $\left.\alpha \mathrm{Neu} 4,5,9 \mathrm{Ac}_{3} 2 \mathrm{Me}\right)(45)$.

Considering MHV-S HE as a useful tool for the histological detection of Neu4,5 $\mathrm{Ac}_{2}$, our collaborators had previously expressed the esterase-inactive MHV-S HE ectodomain as a fusion protein with a C-terminal Fc domain of human IgG1 and investigated $\mathrm{Neu} 4,5 \mathrm{Ac}_{2}$ distribution in human and mouse tissues (46). In another study, we further modified the virolectin by fusing MHV-S-HE ectodomain-Fc to a hexahistidine ( $\left.\mathrm{His}_{6}\right)$ sequence and detected high expressions of 4OAc-Sias in horse and guinea pig respiratory tract tissues than mouse where it was mostly localized in gastrointestinal tract. 4-OAc-Sias were also found in a small number of cells within the duck, dog, and ferret respiratory tissues screened but not so far in the tissues of humans or pigs (47).

To derive a stable 4-OAc-Sia binding probe, we expressed the MHV-S-HE esterase inactive ectodomain (S45A), and the nonbinding mutant MHV-S-HE (F212A), as fusion proteins with the C-terminal Fc domain of human IgG1, along with Avi-tag for permanent biotinylation (Suppl Fig S3). Sia-binding and nonbinding proteins MHV-S-HE protein probes were biotinylated using manufacturer's protocol and tested for their specificity for 4-OAc-Sias on sialoglycan microarray. As anticipated, biotinylated Sia-binding MHV-S-HE (S45A) exhibited very specific recognition of towards 4-OAc-Sias while the nonbinding mutant MHS-S HE (F212A) did not show any binding with any sialylated or non-sialylated glycan on the microarray, confirming its suitability as a non-binding control (Figure 1; Suppl Fig S4). Biotinylated MHV-S-HE (S45A) binds exclusively to $\mathrm{Neu}_{4,5 \mathrm{Ac}} \alpha 3 \mathrm{Gal} \beta 3 \mathrm{GalNAc} \alpha \mathrm{R} 1, \quad \mathrm{Neu} 4,5 \mathrm{Ac}_{2} \alpha 3 \mathrm{Gal} \beta 3 \mathrm{GalNAc} \beta \mathrm{R} 1$, 
Neu4,5Ac $2 \alpha 3$ Gal $\beta 3$ GlcNAc $\alpha R 1 ， N e u 4,5 \mathrm{Ac}_{2} \alpha 3$ Gal $\beta 3$ GlcNAc $\beta R 1 ， N e u 4,5 \mathrm{Ac}_{2} \alpha 3 \mathrm{Gal} \beta 4 \mathrm{Glc} \beta \mathrm{R} 1$, $\mathrm{Neu} 4,5 \mathrm{Ac}_{2} \alpha 3 \mathrm{Gal} \beta 4 \mathrm{GlcNAc} \beta \mathrm{R} 1$ and $\mathrm{Neu} 4,5 \mathrm{Ac}_{2} \alpha 3 \mathrm{Gal} \beta 4 \mathrm{GlcNAc} \beta 3 \mathrm{Gal} \beta 4 \mathrm{Glc} \beta \mathrm{R} 1$ (Figure 1). Despite such high specificity and avidity for Neu4,5 $\mathrm{Ac}_{2}, \mathrm{MHV}-\mathrm{S}-\mathrm{HE}$ (S45A) did not show any binding of 4-OAc-Neu5Gc-glycans on the array (Fig 1). Currently, the sialoglycan microarray does not include $\alpha$ 2-6-linked 4-OAc-Sias (Neu5Ac or Neu5Gc), hence the binding of MHV-S-HE a2-6-linked 4-OAc-Sias is not discussed here.

Considering the rare occurrence of Neu5Gc4Ac and limited knowledge about 4-OAc-Siarecognizing proteins, MHV-S HE-derived fusion proteins provide the most useful probes for in situ detection of 4-OAc-Sias, represented mostly by Neu4,5 $\mathrm{Ac}_{2}$. Given its exclusive preference for 4-OAcetylated-Sias, the biotinylated MHV-S-HE esterase inactive ectodomain appears to be the best probe for this class of sialoglycans (SGRP $4^{M H V}$ and $\mathrm{SGRP} 4^{M H V} \mathrm{NB}$ ) and demonstrate its utility through ELISA, Western blotting, FACS and histochemistry in this article. We expect that SGRP4 ${ }^{M H V}$ will facilitate research on functional significance of 4-OAc-Sias, help finding more comprehensive 4-OAc-Sia binding proteins and will allow us to seek an improved version of SGRP4 with recognition of both Neu4,5 $\mathrm{Ac}_{2}$ and Neu5Gc4Ac.

SGRP5 ${ }^{I g Y}$ recognizes all Neu5Gc-terminated glycans. Humans are genetically defective in synthesizing the common mammalian Sia- Neu5Gc but can metabolically incorporate small amounts of this Sia from dietary sources into glycoproteins and glycolipids of human tumors, fetuses and some normal tissues (48). Neu5Gc derived from dietary sources has been observed in breast, ovarian, prostate, colon and lung cancers. Thus, there is need for sensitive and specific detection of Neu5Gc in human tissues and biotherapeutic products. Previously a number of different monoclonal antibodies against Neu5Gc have been reported which recognized Neu5Gc only in the context of particular underlying sequences, but those generally lack the ability to detect 
Neu5Gc on other structurally related or unrelated glycans (49-55).

We could not find a microbial Sia-binding protein that is completely specific for Neu5Gc, as there is always some cross-reactivity with few Neu5Ac glycans (51-53). Previously, we had improved the specificity and reduced background cross reactivity of a chicken polyclonal antiNeu5Gc-glycan IgY by modifying the affinity purification procedures (56). Since then, this chicken anti-Neu5Gc IgY has been the only available probe for comprehensive in situ Neu5Gcglycan detection.

To explore alternative Neu5Gc-specific probe from microbial sources, we previously reported Neu5Gc-binding preferences of subtilase cytotoxin (SubAB), an AB5 toxin secreted by Shiga toxigenic Escherichia coli (STEC) (10). SubB, the B5 subunits of STEC AB5 toxin, exhibits strong preference for Neu5Gc-terminating glycans and serves as key structural basis of STEC's specificity towards Neu5Gc. SubB showed 20-fold less binding to Neu5Ac; over 30-fold less if the Neu5Gc linkage was changed from $\alpha 2-3$ to $\alpha 2-6$. Neu5Gc-binding by SubB suggested that a more selective diagnostic tool for Neu5Gc detection can be derived from the native protein. Day and colleagues, using molecular modeling and site directed mutations, managed to reduce the $\alpha 2-$ 3 to 2 2-6-linkage preference, while maintaining or enhancing the selectivity of SubB for Neu5Gc over Neu5Ac (57). This SubB analog, SubB2M (SubB $\Delta$ S106/DT107 mutant) did display improved specificity towards Neu5Gc, bound to $\alpha 2-6$-linked-Neu5Gc and could discriminate Neu5Gc- over Neu5Ac-glycoconjugates in glycan microarrays, surface plasmon resonance and ELISA assays. SubB2M also showed promising diagnostic properties in detecting Neu5Gcglycans in early stages of ovarian cancer, and in late-stage diseases which express high levels of Neu5Gc-glycans (58).

Considering the promising Neu5Gc-binding properties of SubB proteins, we attempted to 
derive a comprehensive probe for Neu5Gc-glycans from SubB and tested His 6 -tagged versions of the reported SubB proteins including SubB, SubB2M and SubB2M-nonbinding mutant (SubB2M, S12A) on the glycan array. SubB2M exhibited significantly improved recognition of Neu5Gcglycans compared to the native SubB, while the nonbinding mutant of Sub2M did not show any binding with glycans on the array (Suppl Fig S7). While we were able to reproduce the higher Neu5Gc preferences of Sub2M over native SubB, (59). our sialoglycan microarray data suggested that despite having promising Neu5Gc-binding properties, the Sub2M lacked the scope and robustness of Neu5Gc binding abilities of chicken anti-Neu5Gc IgY (Suppl Fig S7).

Considering results obtained from the Neu5Gc-binding molecules discussed or tested above, it can be inferred that there is no contemporary molecule better than the affinity-purified chicken polyclonal anti-Neu5Gc-IgY to detect a broader range of Neu5Gc-glycans. Hence, we selected chicken polyclonal specific anti-Neu5Gc-IgY as SGRP5 and biotinylated this along with its nonbinding control IgY (from non-immunized chickens) as the final set of SGRP5 ${ }^{\operatorname{IgY}}$ (Suppl Fig S4). The utility of chicken Neu5Gc-IgY as $\mathrm{SGRP}^{\operatorname{Ig} Y}$ in general lab-used methods including ELISA, Western Blotting, FACS and histochemistry is discussed in an earlier article, and the results obtained here confirmed its Neu5Gc-specificity as previously reported $(56,60)$. In a long run, it is necessary to identify a monoclonal $\mathrm{IgY}$ that can detect all forms of Neu5Gc-sialoglycans. SGRP6 $^{S N A}$ recognizes all $\boldsymbol{\alpha 2 - 6 - S i a s . ~ U n l i k e ~ b a c t e r i a , ~ s o m e ~ v i r u s e s ~ t h a t ~ c a u s e ~ u p p e r ~ r e s p i r a t o r y ~}$ infections such as human influenza A, B viruses, and human coronavirus OC43 exhibit preferential affinity towards $\alpha 2-6$ Sias, which are abundant in the upper airway epithelial brush border in humans $(61,62)$. Influenza viral haemagglutinins (HA) are the major glycoproteins that allow the recognition of cells in the upper respiratory tract or erythrocytes by binding to $\alpha 2-6$ Sias, and this property makes them potential candidates for $\alpha 2-6$ Sias-binding probes. We investigated a range of 
viral HAs in the form of HA-Fc fusion proteins (soluble HA fused to human IgG1 Fc) for their sialoglycan-binding specificity using the glycan microarray (Suppl. Fig S8). Among the tested HA-Fc fusion proteins, Cali09 HA-Fc derived from California/04/2009 H1N1 showed selective binding to $\alpha 2-6$-linked Sias, most prominently with Neu5Ac $\alpha 6$ Gal $\beta 4$ GlcNAc $\beta R 5$, followed by Neu5Ac $\alpha 6$ Gal $\beta 4 G$ GlcNAc $\beta R 1$ and Neu5,9Ac $2 \alpha 6$ Gal $\beta 4 G l c N A c \beta R 1$. Despite strong preferences for these $\alpha 2-6$ Sias, none showed binding with a full range of $\alpha 2-6 \mathrm{Sias}$, specially $\alpha 2-6 \mathrm{Neu} 5 \mathrm{Gc}$ glycans. The failure of Cali09-HA-Fc to recognize a number of $\alpha 2-6$-linked sialosides, and its binding to a few $\alpha 2-3$ Sia-linked sialosides questioned its suitability as a probe for $\alpha 2-6$ linked Sias (Suppl. Fig S8). Aichi68-HA-Fc derived from the hemagglutinin of Aichi/2/1968 H3N2 strain also lacked robustness and specificity showing indiscriminate binding towards a number of $\alpha 2-6$ and $\alpha 2-3$ Sias (Suppl. Fig S8). Surprisingly one candidate, PR8 HA-Fc derived from Influenza strain A/Puerto Rico/8/1934 (PR8 H1N1)), even showed prominent binding of $\alpha 2-3$-sialylated glycans instead of $\alpha 2-6$ Sias (Suppl. Fig S8). Among other tested viral haemagglutinins, SC18 HAFc derived from influenza A H1N1 (A/SouthCarolina/1/18) exhibited selective binding to a few 22-6 Sias such as Neu5Ac $\alpha 6$ Gal $\beta 4$ GlcNAc $\beta$ R5 and Neu5Ac $\alpha 6$ Gal $\beta 4 G l c N A c \beta R 1$ while another virolectin Mem-HA-Fc from influenza A H1N1 (A/Memphis/1/1987) bound to $\alpha 2-3$ Sias and $\alpha 2-$ 6Sias without any strong preference for either linkage (Suppl. Fig S8). Taken together, it can be inferred that the viral haemagglutinins tested exhibited promising specificity towards $\alpha 2-6$ Sias but lacked the robustness and binding dynamics required for a probe. Our results and observations appear true for several other viral hemagglutinins not included in this study (63).

As an alternative $\alpha 2-6$ Sia-specific microbial probe, we reviewed the $\alpha 2-6$ Sia-binding properties of a recombinant lectin (PSL) from the mushroom Polyporus squamosus that was reported for its high affinity binding with Neu5Aca2-6Galß4GlcNAc $(64,65)$. However, PSL 
showed a high order preference towards $\alpha 2-6$ over $\alpha 2-3$ Sias but bound exclusively to N-linked recognizing Neu5Aca6GalNAc on serine/threonine-linked glycoproteins, we did not experimentally investigate this molecule further as an $\alpha 2-6$ Sia-specific probe.

As an $\alpha$ 2-6Sia-binding alternative from mammals, we considered the well-characterized vertebrate sialic acid-dependent adhesion molecule CD22/Siglec-2 which is a member of the immunoglobulin superfamily that is expressed by B lymphocytes and binds specifically to Neu5Ac $\alpha 6$ Galß4GlcNAc (66-70). hCD22-Fc (human CD22 fused with the Fe region of human IgG1) showed high affinity for a few $\alpha 2-6$ Sias on microarray (Suppl Fig S9), but lacked the avidity required for a probe, particularly for $\alpha 2-6$-linked Neu5Gc. With a possibility to characterize 2 SGRP6 candidates; one specific for $\alpha 2-6 \mathrm{Neu} 5 \mathrm{Ac}$ and the other for $\alpha 2-6 \mathrm{Neu} 5 \mathrm{Gc}$, we tried exploiting the evolutionary derived strong preference of mouse siglec-2 (mCD22) for $\alpha 2$ 6Neu5Gc. mCD22-hFc showed exclusive binding towards $\alpha 2-6 \mathrm{Sias}$, but preferred Neu5Gc in general (Suppl Fig S9). Interestingly, 9-O-acetylation completely aborted hCD22's binding to $\alpha 2-$ 6Neu5Ac (Suppl. Fig S9) but did not affect mCD22 binding to $\alpha 2-6$ Sias. Nevertheless, the relatively poor avidity and inability of hCD22 to recognize O-Ac-substitution in $\alpha 2-6 \mathrm{Neu} 5 \mathrm{Ac}$, and the non-specificity of $\operatorname{mCD} 22$ binding $\alpha 2-3$ sialyl-LNnT glycan (Neu5Gc $\alpha 3$ Gal $\beta 4 G l c N A c \beta 3 G a l \beta 4 G l c)$ underlined their incompetency as an ideal $\alpha 2-6$ Siabinding probes, even in combination (Suppl Fig S9).

Among other molecules, we also analyzed the adhesin of non-typeable Haemophilus influenzae which was reported for high affinity towards $\alpha 2-6$-linked Neu5Ac (71). According to this study, HMW2 bound with high affinity to $\alpha 2-6$-linked Neu5Ac such as Neu5Ac $\alpha 6$ Gal $\beta 4$ GlcNAc ( $\alpha 2-6$-sialyllactosamine) and could discriminate it from $\alpha 2-3$ Sias 
( $<120$-fold lesser binding with $\alpha 2-3$ Siallylactosamine).-linked siallylactosamine). HMW2 indeed showed appreciable preference for the $\alpha 2-6$ over the $\alpha 2-3$-linkage but was not able to recognize $\alpha 2-6 \mathrm{Neu} 5 \mathrm{Gc}$, disqualifying it from consideration as a comprehensive probe for $\alpha 2-6 \mathrm{Sias}$. In the absence of an evolutionarily derived microbial protein as a dynamic probe for $\alpha 2-6$ Sias, we considered whether SNA (Sambucus nigra or elderberry bark lectin) is still the best available probe for $\alpha 2-6$ Sias.

SNA exhibits high preference for the terminal Neu5Ac $\alpha 6 \mathrm{Gal} / \mathrm{GalNAc}$ sequences in both N-linked and O-linked glycans (72). Due to its ability to discriminate Siaa6Gal/GalNAc from Sian2-3Gal/GalNAc and ubiquitous binding to Neu5Ac/Gc with/without O-Ac substitutions, SNA has been the standard probe for studying $\alpha 2-6$ Sias. Using Vector lab's Biotin-SNA, we investigated SNA's Sia-binding efficiency in sialoglycan microarray and observed very high preference towards $\alpha 2-6 \mathrm{Sias}$ for both Neu5Ac and Neu5Gc and no measurable binding to $\alpha 2-$ 3Sias (Fig 1). Interestingly, the $O$-acetyl substitutions that were a major concern for hCD22, mCD22, viral hemagglutinins and HMW2, did not hinder SNA's binding to $\alpha 2-6$ Sias. Apart from its binding to 6-O-sulfated Gal $\beta 4 \mathrm{GlcNAc}(73)$, SNA displayed an exclusive preference for $\alpha 2-$ 6Sias in disaccharides, trisaccharides and oligosaccharides in Asn or Ser/Thr-linked glycans. There appears to be no better molecule than SNA to probe $\alpha 2-6$ Sias, hence we selected Biotin-SNA as SGRP6 (SGRP6 ${ }^{S N A}$ ) for this study. SGRP6 ${ }^{S N A}$ is the exception to the set of SGRPs in not having an internal control of specificity. We characterized SGRP6 $6^{S N A}$ as $\alpha 2-6$ Sia-probe without any nonbinding variant in routine lab assay methods in this article.

SGRP7 ${ }^{B C o V}$ recognizes an exclusive class of 7,9-diOAc-Sias. Except for some claims of 7-Omono-acetylated Sias in microorganisms $(74,75)$ and on human lymphocytes $(76)$, 7-OAc-Sias are not commonly reported in natural glycans because of their instability. During synthesis, the 
primary attachment site of $O$-acetyl groups was exclusively to the hydroxyl at $\mathrm{C}-7$ of Sias, and the ester group migrates from C-7 to C-9 (77). A hypothesis was proposed that SOAT enzyme would effectively be a 7-O-acetyltransferase incorporating O-acetyl groups primarily at $\mathrm{C}-7$ of sialic acids, followed by their migration to the primary hydroxyl group at C-9 and further transfer of the acetyl residue to $\mathrm{C}-7$, resulting in di- and tri- $O$-acetylated species (78). This suggests that in nature 7-OAc will be represented as 7,9-diOAc or 7,8,9-triOAc, but largely as 7,9-diOAc in mammalian sialoglycans. Considering, there is no stable 7-OAc, there may not be a true 7-OAc-Sia-binding protein, we defined specificities of SGRP7 to 7,9-diOAc-Sias. 7/9di,9-diOAc has traditionally been studied in bovine submaxillary mucin, where the amount of 7-OAc- and 7,9-diOAc-Sias combined is almost twice of 9-mono-OAc-Sias (46).

We also reviewed the sialoglycan specificity of lectins and microbial proteins, previously reported for their binding to 7,9-diOAc-Sias. Lectins from Cancer antennarius, Achatina fulica and Tritichomonas foetus showed higher preferences for 9-OAc and somewhat to 4-OAc but negligible to poor affinities for 7,9-diOAc-Sias $(33,34,79)$. Viruses exhibit prominent binding to O-Ac-Sias but most show either 9-OAc preference (Influenza C viruses, human coronavirus OC43, porcine encephalomyelitis virus) or 4-OAc preference (Infectious salmon anemia virus, Puffinosis virus, mouse hepatitis virus strains-S, DVIM, JHM) binding over 7,9-diOAc $(35-40,46,80,81)$. The hemagglutinin esterases from bovine toroviruses (BTOV-B150, BToV-Breda), bovine coronaviruses (strains; Mebus and Lun) and equine coronavirus (ECoV-NC99) preferentially cleave 7,9-diOAc substrates over mono 4- or 9-OAc-substrates $(46,82)$. Bovine viruses exhibit selective binding towards 7,9-diOAc, particularly BCoV-Mebus having relatively pronounced preferences towards 7,9-diOAc variants of both Neu5Gc and Neu5Ac, but lower preferences for 9-OAcs (46). It is interesting that BCoV esterase selectively removes all 9-OAc residues in 7,9- 
diOAc-Sias in BSM, leaving 7-OAc attached. The residual 7-OAc residues do not attract binding of $\mathrm{BCoV}$ anymore, demonstrating that $\mathrm{Sia} 9-\mathrm{O}$-acetylation is a strict requirement and that mono 7-OAc-Sias do not serve as ligands (46). The S protein, another hemagglutinin in BCoV, showed high specificity exclusively for 9-OAc-Sias with no preference for 7-OAc and this raises possibility that $\mathrm{HE}$ is the only receptor-binding protein for 7,9-di-Ac in BCoV-Mebus $(83,84)$.

Since BCoV-Mebus-HE is the best characterized for 7,9-diOAc-Sia specificities, we expressed its esterase inactive mutant (BCoV-Mebus-HE S40A) and corresponding non-binding mutant (BCoV-Mebus-HE F211A), each fused to human IgG-Fc followed by a His 6 -tag and an Avi-tag. Both proteins were biotinylated with an Avi-tag and were tested for their sialoglycan preferences on sialoglycan microarray (Fig 1, Suppl Fig S4). As observed, the nonbinder mutant does not show any binding with any sialylated or non-sialylated glycans in the array while the Siabinding molecule BCoV-Mebus-HE S40A exhibited some affinity for 9-OAc-Sias (Fig 1), agreeing with its Sia-binding pattern on sialoglycan microarray reported previously (46). However, similarly encouraging as the BCoV-Mebus-HE, S40A did not exhibit any binding to 4-OAc, most 9-OAc-Sias and non-acetylated sialyloligosaccharides, the sialoglycan microarray did not contain 7/9diOAc,9-diOAc-Sias, so it is not known whether the protein binds that form.

SGRP8 $^{T e T}$ binds to major class of ( $\alpha 2-8$-linked) di-Sia oligosaccharides. Oligosaccharide sequences in gangliosides and a few glycoproteins that terminate in Sias, are not always monosialylated. In addition to $\alpha 2-3$ or $\alpha 2-6$-linkage with penultimate Gal, Sia can be linked with another Sia predominantly by $\alpha 2-8$-linkage forming $\alpha 2-8$-linked disialosides, $\alpha 2-8$ linked oligosialic and a2-8-linked polysialic acid chains. $\alpha 2-8$-linked disialyl structures are critical component of neuronal gangliosides and these disialyl gangliosides, specially GD3 and GD2 have been utilized as tumor markers for melanomas, gliomas and neuroblastomas $(28,30-32)$. Due to their utility in 
cancers either as biomarker for in vivo immunolocalization or in phase I and II trials to target disseminated neuroblastoma, a range of anti-disialoganglioside monoclonal antibodies have also been generated. For example, R24 is a mouse IgG3 monoclonal antibody (MAb) that reacts with the ganglioside GD3 expressed by cells of neuroectodermal origin (85). Similarly, a list of antiGD2 specific MAbs; 3F8 (86), BW704 (87), 14G2a, 15G2a (88), AI-201, AI-287, AI-410. AI-425 (89) and anti-GD3 antibodies like MAbs AI-245 and AI-267 (89), Ch14.18 and Ch14.18/CHO have been characterized for their preferential binding to disialyl gangliosides. Further, MAbs JONES, D1.1 and 27A showed affinity for 9-OAc-GD3 but not 9-OAc-GD2 while Mab 8A2 binds both (90). Despite the large number of GD3 and GD2 MAbs, there has not been a single MAb that could serve as a comprehensive probe for the broader range of disialylated glycans.

Among mammalian proteins, myelin-associated glycoprotein (MAG) shows high binding for specific gangliosides in order of GQ1b $\alpha>$ GT1a $\alpha$, GD1 $\alpha>$ GD1a, GT1b $>>$ GM3, GM4, but not for GM1, GD1b, GD3, and GQ1b. Despite high affinity for disia and oligosias, MAG's binding to gangliosides is not exclusive for di-Sia linkages $(91,92)$. Siglec-7 is also reported to react mainly with disialyl structures such as disialyl Lewis $\alpha$, disialyl galactosyl globoside, and ganglioside GD3, although it also lacks the probe like binding dynamics for this class (93). Among lectins reported to bind to disialylated oligosaccharides, few are Cancer antennarius lectin, Agrocybe cylindracea lectin and WGA. Cancer antennarius lectin binds with GD3 (94), Agrocybe cylindracea lectin binds with GDla and GD1b while not clearly differentiating other structures

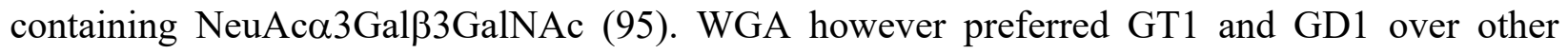
gangliosides, exhibits very high affinity for monosialylated glycans and GlcNAc also, hence cannot be used as a di-Sia linkage specific probe (96).

Infectious microbes possess promising affinity for disialyl oligosaccharides in accordance 
with the glycan environment of their anchorage surfaces. Porcine sapelovirus (PSV) binds specifically to GD1 $\alpha$ (97) and Helicobacter pylori recognizes GD2, GD1 $\alpha$ and GD1 $\beta$ by its sialic acid-binding adhesin (SabA) (98). In agreement with high affinity for single ganglioside as observed in Vibrio cholerae (GM1), the majority of gut infecting bacteria and viruses exhibit binding with a specific sialoglycan structure, and their affinity cannot be generalized for range of disialyloligosaccharide sequences.

Tetanus (TeT) and botulinum neurotoxins $(\mathrm{BoT})$ are produced by anaerobic bacteria Clostridium tetani and Clostridium botulinum, respectively, and share significant structural and functional similarity (99). Not only BoT (serotypes A to G) and TeT exhibit affinities for similar di and oligosialogangliosides but also share significant sequence homology. In both BoT and TeT, $150 \mathrm{Kda}$ single chain can be cleaved in to $50 \mathrm{Kda} \mathrm{N}$-terminal light chain and $100 \mathrm{Kda} \mathrm{C}$-terminal heavy chain. The HC fragment plays the primary role in receptor binding and can be further cleaved into $50 \mathrm{Kda} \mathrm{N}$-terminal fragment $(\mathrm{HN})$ and $50 \mathrm{Kda} \mathrm{C}$-terminal fragment $(\mathrm{HC})$. $\mathrm{HC}$ domain or HCR is characterized by the amino acid sequence homology among BoTs and TeT, which suggests that a conserved amino acid motif of in this domain may define a common carbohydrate recognition site. Since both BoT and TeT possess comparable binding specificity for gangliosides, we preferred TeT to investigate its potential for a comprehensive disialyl linkage recognizing probe. Selection of $\mathrm{TeT}$ over BoT was also based on detailed reports on TeT binding to gangliosides (100-103), and feasibility to select single serotype of TeT than BoT that has multiple serotypes. An optimal receptor-binding domain (residues 1105 -1315) from TeT-HCR was expressed as SNAPf-His6 fusion in the pGEX-3X vector in a bacterial expression system along with its mutants TeT-HCR R1226L, TeT-HCR W1289A, and TeT-HCR R1226L/W1289A (Suppl Fig S3 \& S10). Expressed proteins were biotinylated by SNAP-Biotin chemistry as described in 
experimental procedures and tested for their sialoglycan affinity in microarray (Fig 1, Suppl Fig S4, S10). TeT has been shown to specifically bind gangliosides of the G1b series, GD1b or GT1b. In accordance with published report (102), TeT-HCR bound with a range of sialogangliosides including monosialylated GM1 $\alpha$, fucosyl-GM1 and oligosialylated GD1 $\alpha$, GD3, GT3 (Suppl Fig S10). The high affinity of TeT-HCR for GM1 and Fuc GM1 questioned its candidacy as di-Sia linkage specific probe. Single mutant; TeT-HCR R1226L and double mutant TeT-HCR R1226L/W1289A didn't bind with any glycans on array, suggesting the significance of arginine residue at 1226 position in sialoglycan identification (Suppl Fig S4 and S10). Another single mutant TeT-HCR W1289A showed appreciable enhancement in affinity towards GD3, GT3 while loss in binding towards monosialylated and asialylated glycans on array (Suppl Fig S10). With respect to TeT-HCR (native protein), the tryptophan mutant TeT-HCR W1289A had pronounced specificity towards disialogangliosides structures particularly Neu5Ac $\alpha 8$ Neu5Aca3Gal $\beta 4$ Glc $\beta R 1$, Neu5Ac $\alpha 8$ Neu5Ac $\alpha 3$ Gal $\beta 4 G l c \beta R 4, \quad$ Neu5Ac $\alpha 8 N e u 5 A c \alpha 8 N e u 5 A c \alpha 3 G a 1 \beta 4 G 1 c \beta R 4, \quad$ and

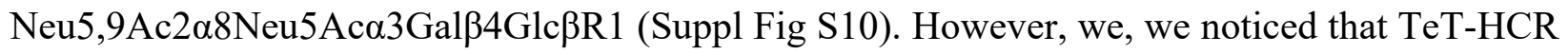
W1289A exhibited some minor binding with monosialogangliosides on the array but based on its high affinity for the range of $\alpha 2-8$-linked disialyl oligosaccharides and lack of other available options (MAbs, plant lectins, siglecs, viral, bacterial proteins) to derive a more potent probe for this class, we selected biotinylated TeT-HCR W1289A as SGRP8 (SGRP8 ${ }^{T e T}$ ). We selected the double mutant; Biotinylated TeT-HCR R1226L/W1289A (SGRP8 ${ }^{T e T} \mathrm{NB}$ ) as a nonbinding control of SGRP8 ${ }^{T e T}$ due to its complete loss of binding for any glycans on array. The broad specificity of several bacterial toxin including TeT-HCR towards GM1, GD3, GT3 and GQ series of glycans could be related with evolutionary preference to bind with most available ligands in their neural niche. This nonexclusive binding with multiple ligands by toxins might have advantage for the 
microbes but reduces our possibility to derive a molecule with probe like robustness and dynamic specificity. Here, we present that site-specific modifications could improve the binding range in otherwise 'nonspecific' probe candidates and characterize SGRP8 ${ }^{T e T}$ as $\alpha 2-8$-disiaoligosaccharide binding probe through general laboratory methods later in this report.

SGRP9 $^{P T O V}$ is a competent probe for 9-OAc-Sias. Of the variety of O-Ac-Sia modifications in nature, 9-O-acetylation is the most common in cells and tissues of humans and other animals. Their distribution is highly variable and implicated in embryonic development, host pathogen interactions and immunity. Although there have been monoclonal antibodies against $O$-acetylated gangliosides, these MAbs remained highly specific in recognition of $O$-acetyl esters only when represented by specific underlying sugar chain (104). Few Sia specific lectins recognizing 9-OAc modifications have been reported, for example a lectin from the marine crab Cancer antennarius showed affinity for 9-OAc and was used to demonstrate the presence of tumor-associated 9-OAcGD3 on human melanoma cells (33). Cancer antennarius lectin also showed significant affinity for Neu4,5Ac2, and didn't show affinity for 9-OAc. AchatininH, a lectin from the hemolymph of African land snail Achatina fulica did not bind to Neu4,5Ac2, but failed to exhibit probe-like preference for a number of 9-OAc-Sias (105). Another lectin from protozoan, Tritichomonas foetus showed promising affinities for 9-OAc-Sias but also bound to de- $O$-acetylated Sias relatively high affinity, confirming its unsuitability as a probe (34).

9-OAc-Sia-binding specific influenza C virus hemagglutinin esterase (Inf-CHE) was previously utilized as whole virions or recombinant protein for assessing a wide spectrum of sialoglycoconjugates like mucins, serum glycoproteins or gangliosides containing naturally or synthetically $O$-acetylated sialic acids (106). The influenza $\mathrm{C}$ virus hemagglutinin-esterase is a membrane-bound glycoprotein that binds specifically to 9-O-acetylated sialic acids 
(hemagglutinin activity) and then hydrolyzes the $O$-acetyl group (receptor-destroying activity). Inf-CHE can specifically cleave O-acetyl groups from $\mathrm{Neu} 5,9 \mathrm{Ac}_{2}$ but not from $7-\mathrm{O}$-acetyl- $\mathrm{N}$ acetylneuraminic acid $\left(\mathrm{Neu} 5,7 \mathrm{Ac}_{2}\right)$, and very slowly from $\mathrm{Neu} 4,5 \mathrm{Ac}_{2}$ (107). Previously, we demonstrated that inactivation of Inf-CHE esterase by treatment of serine esterase inhibitor di-isopropyl fluorophosphate (DFP) resulted into a stable and irreversible binding with 9-OAcSias (106). However, using the whole virion as a probe was complicated due to variations in purity and stability of preparations, poor reproducibility, high nonspecific background, and lack of linearity in response. To avoid these limitations, we had replaced Inf-C virions with a soluble and recombinant chimeric molecule composed of the extracellular domain of Inf-CHE fused to the Fc region of human IgG1 (CHE-Fc). DFP inactivation of CHE-Fc stabilized the hemagglutinin activity and yielded a probe $\mathrm{CHE}-\mathrm{FcD}$ that was more specific for 9-OAc-Sias than the whole InfC virion $(108,109)$. CHE-FcD provided a better alternative but did not qualify as a standard probe for 9-OAc-Sias due to selective preference for Neu5,9Ac 2 over Neu9Ac5Gc glycans, nonspecific binding to Neu5,7 $\mathrm{Ac}_{2}$ and hazards related to use of DFP (Supp. Fig S11), (40).

In order to derive a better probe for 9-OAc-Sias, we reviewed other O-Ac-esterases from Inf-C like mammalian coronaviruses; bovine (bovine coronaviruses; BCoV strains- Mebus, LUN, Breda, B150), birds (puffinosis virus), human (human coronaviruses, strains-OC43, HKU1), equine (ECoV-NC99), murine (mouse hepatitis virus strains-S, DVIM, JHM) and porcine (porcine torovirus strains; PToV, strains- Markelo, P4), reported previously (46, 80, 81). Based on these reports, which also includes our previous studies on OAc-Sia specificity of nidovirus HEs, we selected Porcine Torovirus P4 strain (PToV) hemagglutinin esterase to investigate as a potential probe for this class. Following the approach that we used for CHE-Fc, we expressed PToV HE ectodomain in HEK293T cells as fusion proteins with a C-terminal Fc domain of human IgG1 
(PToV-HE-Fc). Instead of using DFP, we used site-directed mutagenesis (S46A) to inactivate the esterase activity in PToV-HE-Fc fusion protein. PToV-HE-Fc showed high selectivity towards 9OAc-Sias and demonstrated its applicability to revealing the OAc-Sia distribution in human and animal tissues and cell lines $(46,47)$.

We expressed 9-OAc binding protein (PToV-HE-Fc, S46A) and nonbinding protein (PToV-HE-Fc, F271A) fused to human IgG-Fc followed by a His6 tag and an Avi-tag (Suppl. Fig S3). Both binding and nonbinding proteins were biotinylated using the Avi-tag and investigated for their affinities in a sialoglycan microarray. The esterase-inactive probe; PToV-HE-Fc (S46A) bound exclusively with 9-OAc-Sias including Neu5Ac and Neu5Gc, while hemagglutinin inactive nonbinding PToV-HE-Fc (F271A) did not show affinity towards any sialylated or nonsialylated glycans on array (Fig 1, Suppl Fig S4). PToV-HE-Fc (S46A) showed binding towards Neu5Ac and Neu5Gc $\alpha 2-3, \alpha 2-6$ and $\alpha 2-8$-linked to their penultimate sugars. In a similar sialoglycan microarray, CHE-FcD showed efficient binding with a number of Neu5,9Ac 2 -glycans but did not show similar affinity for Neu5Gc9Ac and remained a relatively weaker binder to 9-OAc-Sias in general (Suppl Fig S11). Alternatively, PToV-HE-Fc (S46A) exhibited significantly stronger binding towards a wide range of 9-OAc-Sia-glycans in comparison with CHE-FcD. Taking together, the efficacy, exclusivity and reproducibility of PToV-HE-Fc (S46A) as 9-OAc-Siabinding protein, we selected biotinylated PToV-HE-Fc (S46A) as comprehensive probe (SGRP9 ${ }^{P T o V}$ ) for 9-OAc-Sia and biotinylated nonbinding PToV-HE-Fc (F271A) as SGRP9 ${ }^{P T o V}$ NB, the internal control of SGRP9 ${ }^{\text {PToV' }}$ 's specificity. We characterize the utility of PToV-HE-Fc (S46A) as SGRP9 ${ }^{P T O V}$ in general laboratory methods in sections later in this article.

Use of the optimized panel of SGRPs in common methods. Although the detailed sialoglycan microarray analysis provides insights into the Sia specificity of SGRPs, it is essential for SGRPs 
to exhibit equally precise affinities towards their ligands in ensemble of glycans represented in biological samples. In particular, the multi-antennary sialoglycans and structurally overlapping sialoglycans in biological samples may have a different interactions with probes than observed with individual sialoglycans in the glycan arrays. Qualitative Sia binding and ligand specificity of SGRPs were therefore tested by ELISA experiments with blood sera from 9 animal species, each signifying diverse sialoglycan composition in terms of presence or absence of N-glycolyl at C5 of Sia, $O$-acetylation, types of glycosidic bonds to penultimate sugars and overall oligosaccharide sequence. For example, mouse serum is proportionately high in Neu5Gc-Sias in comparison with $\mathrm{Cmah}^{-/-}$mouse that remain exclusive for Neu5Ac-Sias and relatively higher in $O$-acetylation. Horse and guinea pig sera were included for their high representation of 4-OAc-Sias which was absent in other sera, as observed in HPLC analysis of Sias (Suppl. Table-1). Similarly, we included erythrocytes from similar 9 animal species, expecting to derive a relatable pattern of SGRPs binding with sera and RBCs. HPLC analysis of surface sialome of erythrocytes (Suppl. Table-2) suggests high variance in Sia diversity among animals which would be interesting to detect with SGRPs. We emphasized on minimal loss of heat and pH labile O-Acs during assays and modified the procedures accordingly.

\section{SGRP1 ${ }^{\text {YenB }}$ exhibits Sia specific binding to all mammalian sera.}

As results summarized in Fig 2 suggest, $\mathrm{SGRP} 1^{\text {YenB }}$ exhibits comparable affinity to all sera, indicating its broad range of Sia specificities. In accordance with the observation from glycan array (Fig. 1), SGRP1 ${ }^{\text {YenB }}$ exhibits strong binding towards sera whether it was Neu5Gc rich (mouse, goat) or Neu5Ac abundant (human, rat and $\mathrm{Cmah}^{-/}$mouse). Further, high proportions of Sia modifications such as 9-OAc substitutions (rat, rabbit and $\mathrm{Cmah}^{-/-}$mouse) and more prominently, 4-OAc substitutions (guinea pig and horse serum) didn't inhibit SGRP1 ${ }^{\text {YenB }}$ 's binding to serum 
sialoglycans (Fig. 2A). Sialoglycan microarray (Fig. 1) confirms SGRP1 ${ }^{\text {YenB}}$ 's strong binding towards sialoglycans that may directly be responsible for its binding to immobilized sera, but to exclude the potential contribution of serum protein interactions with SGRP1 ${ }^{\text {YenB }}$ from our observations, we performed binding assays with sera after partial and complete Sia depletion. Mild periodate oxidation of sera followed by borohydride reduction generates side-chain truncated-Sias with a terminal hydroxyl at $7^{\text {th }}$ carbon. SGRP1 $1^{\text {YenB }}$ showed significantly reduced binding to periodate oxidized sera that was proportional to the loss of desired Sia ligands after the truncation (Supp. Fig S12A). A question remained as to whether the residual binding of SGRP1 ${ }^{\text {YenB }}$ to sera, observed even after periodate treatments were due to SGRP1 ${ }^{\text {YenB }}$ interaction with non-sialylated structures in serum, or the $O$-acetyl substitutions protecting Sias from complete oxidation. When $O$-acetyl esters were removed by mild base treatment before periodate oxidation, the residual SGRP1 ${ }^{\text {YenB }}$ binding was completely abolished, thereby excluding the role of protein-protein interaction in SGRP1 ${ }^{\text {YenB }}$ 's binding to mammalian sera (Supp. Fig S12A). In the same experiment, base treatment without periodate oxidation did not significantly influence SGRP1 ${ }^{\text {YenB }}$ binding to sera, confirming the probe's unbiased preference for Sias as seen in glycan array also (Fig 1). Significantly, the nonbinding variant of the probe, SGRP $1^{\text {YenB }}$ did not show any binding with any serum at all tested concentrations, as expected (Fig 2A).

SGRP2 ${ }^{P l t B}$ exhibits Neu5Ac-specific binding to mammalian sera. SGRP2 ${ }^{P l t B}$ binds to Neu5Ac and its derivatives that constitute a major fraction of Sia population in mammalian sera. Accordingly, SGRP2 ${ }^{P l t B}$ exhibited binding to all serum types tested in a concentration dependent manner (Fig 2B). However, SGRP2 ${ }^{\text {PltB }}$ exhibits an interesting pattern of binding towards Neu5Gc rich mouse serum vs Neu5Ac rich sera, especially human and $\mathrm{Cmah}^{-/-}$mouse sera. The non-binding mutant SGRP2 ${ }^{P l t B}$ NB failed to bind serum from any animal source. Binding assays with mild base 
and periodate-treated sera confirmed that SGRP2 ${ }^{P l t B}$ 's binding towards serum was exclusively to Sia and was devoid of nonspecific interactions (Suppl Fig. S12B). The SGRP2 ${ }^{\text {PltB }}$ also exhibited residual binding after periodate oxidation that were further reduced with prior base treatments, but base treatment alone did not noticeably influence the SGRP2 ${ }^{\text {PltB }}$ binding to sera.

\section{SGRP3 $^{H s a}$ exhibits $\alpha 2-3$ Sia-specific binding to mammalian sera.}

SGRP3 ${ }^{H s a}$ is specific towards Sias linked to underlying glycans with $\alpha 2-3$-linkage that coexist with $\alpha 2-6$ Sias and $\alpha 2-8$-linked Sias in cells, tissues, and other biological samples. Among the sera employed to investigate SGRP3 ${ }^{H s a}$ binding towards $\alpha 2-3$-linked Sias in complex biological samples, SGRP $3^{H s a}$ bound all sera in a concentration dependent manner. In a different experiment to test Sia-specific binding of SGRP3 $3^{H s a}$ to serum, the probe exhibited sera binding directly proportionate to their Sia constituents (Suppl Fig S12C), excluding non-specific interaction with nonsialylated structures in serum. Collectively, the results demonstrate SGRP3 ${ }^{\mathrm{Hsa}}$ 's indiscriminate binding to $\alpha 2-3 \mathrm{Sia}$ in serum, irrespective of $O$-acetylation, Neu5Ac or Neu5Gc, a finding that agrees with our observations from sialoglycan microarray (Fig 1). SGRP3 ${ }^{H s a} \mathrm{NB}$, the nonbinding version of SGRP $3^{H s a}$ showed no binding with any serum at tested concentrations (Fig $2 \mathrm{C}$ ).

\section{SGRP4 $^{M H V}$ exhibits 4-OAc-Sia-specific binding to mammalian sera.}

SGRP4 ${ }^{M H V}$ represents a probe for an exclusive class of 4-O-acetylated sialoglycans, which show a high abundance in the blood components and tissues of certain animals. In our collection of sera, 4-OAc is represented by guinea pig and horse $(38.7 \%$ and $32.4 \%$ of total Sia content respectivelySuppl. Table 1) and accordingly SGRP4 ${ }^{M H V}$ exhibited strong affinity towards these sera (Fig 2D). The negligible binding of SGRP $4^{M H V}$ to other sera suggests that its specific binding to guinea pig and horse serum was due it's binding to 4-OAc-Sias exclusively found in these samples. Importantly, SGRP4 ${ }^{M H V}$ exhibited no binding to serum rich in 9-OAcs ( $\mathrm{Cmah}^{-/-}$, rat and rabbit). 
confirming its ability to detect 4-OAcs over 9-OAcs. In similar experiment, SGRP4 ${ }^{M H V} \mathrm{NB}$ didn’t bind to any serum at tested concentrations, confirming its utility as nonbinding control. In another qualitative approach to test SGRP4 ${ }^{M H V}$ 's specificity towards 4-OAcs, its binding was assessed with sera, pretreated with mild base and periodate. Periodate treatment did not have much effect on SGRP4 ${ }^{M H V}$ 's binding towards guinea pig and horse serum, whereas base treatment and base treatment followed by periodate oxidation completely blocked Sia detection (Suppl Fig S12D). These results, along with sialoglycan glycan microarray confirms that even in complex biological samples like serum, SGRP4 ${ }^{M H V}$ binds only to 4 OAcSias

\section{SGRP5 ${ }^{I g Y}$ exhibits Neu5Gc-specific binding to mammalian sera.}

Similar to SGRP4 ${ }^{M H V}$, sera binding experiment for $\mathrm{SGRP}^{\operatorname{Ig} Y}$ had internal indicators of probe' specificity. While $5 \mathrm{~N}$-glycolylated Sias (Neu5Gc) are the major component of Sia diversity in mammals, Neu5Gc is absent from humans due to loss of functional $\mathrm{Cmah}$, and also in $\mathrm{Cmah}^{-/-}$ mice. The serum binding assay (Fig. 2E) exhibits that $\operatorname{SGRP}^{\operatorname{Ig} Y}$ showed very strong affinity for the WT mouse serum, and also binding to all sera except human and $\mathrm{Cmah}^{-/-}$mouse. Significantly $\mathrm{SGRP}^{\operatorname{Ig} Y}$ showed the reverse binding pattern to that was observed with $\mathrm{SGRP} 2^{P l t B}$ (Fig 2B), suggesting an approach to double-check the specificity of such complimentary probes. SGRP5 ${ }^{\operatorname{Ig} Y} \mathrm{NB}$ didn't bind any serum tested in similar experiment, as anticipated. A binding assay with base- and periodate-treated sera supported the specificity of SGRP5 ${ }^{\operatorname{Ig} Y}$, as removal of OAc esters did not affect its binding to serum, while removal of Sias abolished binding (Suppl Fig S12E).

\section{SGRP6 $^{S N A}$ exhibits $\alpha 2-6$ specific binding to mammalian sera.}

SGRP6 ${ }^{S N A}$ exhibits strong and general binding with all mammalian sera in binding experiment, suggesting its robustness as $\alpha 2-6$ Sia-recognition probe. As observed in glycan array (Fig1), 
SGRP6 $^{S N A}$ does not discriminate between $\alpha 2-6 \mathrm{Neu} 5 \mathrm{Ac}$ and $\alpha 2-6 \mathrm{Neu} 5 \mathrm{Gc}$, with or without OAc esters. Similarly, the serum binding data (Fig $2 \mathrm{~F}$ ) shows binding of SGRP6 ${ }^{S N A}$ to mouse, rabbit, guinea pig, human or $\mathrm{Cmah}^{-/}$mouse sera which contain Neu5Gc, Neu5Ac \& Neu5,9Ac, $\mathrm{Neu} 4,5 \mathrm{Ac}_{2}, \mathrm{Neu} 5 \mathrm{Ac}, \mathrm{Neu} 5 \mathrm{Ac}$ and Neu5,9Ac 2 respectively. SGRP6 ${ }^{S N A}$ has traditionally been used as $\alpha 2-6$ Sia recognizing probe, and our binding experiment with periodate oxidized serum confirms that SGRP6 ${ }^{S N A}$ binds to sera in Sia specific manner and does not bind serum proteins (Suppl Fig S12F).

\section{SGRP7 ${ }^{B C o V}$ exhibits 7,9-diOAc-Sia specific binding to Bovine Submaxillary Mucin.}

SGRP7 ${ }^{B C o V}$ exhibited insignificant binding to tested mammalian sera, likely due to very strong preference towards 7,9 diOAcSias, absent in these sera (Fig 2G). The probe does possess inconsistent and minor binding preferences towards 9-OAc-Sias but those were not detected in the sera sialoglycans. To confirm SGRP7 ${ }^{B C o V}$ 's recognition of 7, 9-diOAc-Sias, we performed binding experiments with immobilized BSM, enriched in di- and tri-OAc-sialoglycans. As anticipated, SGRP7 ${ }^{B C o V}$ showed dose-dependent binding patterns to BSM, while the nonbinding mutant SGRP7 ${ }^{B C o V}$ NB showed no detectable signals (Fig 2J). We confirmed its specificity towards OAcSiasOAc-Sias in base and mild periodate-oxidized BSM. The data (Suppl Fig S12G) explains that SGRP7 ${ }^{B C O V}$ binds BSM in OAc-dependent manner, and after depletion of OAc esters resulted in the dramatic reduction in SGRP7 ${ }^{B C o V}$ 's binding, comparable to loss of binding after complete depletion of Sias.

\section{SGRP8 $^{T e T}$ exhibits DiSia linkage specific binding to gangliosides.}

SGRP8 ${ }^{T e T}$ exhibits very specific binding towards di-Sia linkages, the sialoglycan moieties mostly represented by gangliosides in biological system. As a result, probe SGRP8 ${ }^{T e T}$ showed no detectable binding with tested against sera in ELISA experiment (Suppl. Fig S13A). In an ELISA 
experiment with immobilized purified gangliosides, SGRP8 ${ }^{T e T}$ showed dose dependent binding towards di-sia oligosaccharide (GD1, GD3, GT1) discriminating from the mono-sia oligosaccharides (GM1, GM3) and polysia oligosaccharides conformations (Colominic acid) (Fig $2 \mathrm{H})$. The observed results from ganglioside binding experiments are in complete agreement with the sialoglycan microarrays performed with SGRP8 ${ }^{T e T}$ and confirms the advantage of the probe over native TeT-HCR (Fig 1, Suppl. Fig S10 A \& B). As anticipated, the nonbinding mutant SGRP $8^{T e T} \mathrm{NB}$ showed no detectable signals, signifying its utility as control for SGRP8 ${ }^{T e T}$ 's specificity (Fig 2H).

\section{SGRP9 ${ }^{P T o V}$ exhibits 9-OAc-Sia-specific binding to mammalian sera.}

SGRP9 ${ }^{P T o V}$ binds to 9-OAc-Sias that are represented by all mammalian sera included for binding assays (Suppl Table-1). In observed results, SGRP9 ${ }^{P T O V}$ demonstrated remarkably high affinity towards rat and $\mathrm{Cmah}^{-/-}$mouse serum, suggesting their high proportions of Neu5,9Ac $\left(\mathrm{Fig}_{2} \mathrm{I}\right.$ ). However, relatively lower binding to rabbit serum, as detected by signals in ELISA experiment was interesting as rabbit serum contained the highest fraction of $\mathrm{Neu} 5,9 \mathrm{Ac}_{2}$ among the tested sera, but the basis of such variance is not known. It is clear from the serum-binding data of the SGRPs that those can demonstrate the presence or absence of the preferred ligand, but that this may not always measure the proportions relative to other Sia types within the serum. No detectable signals from similar binding assay with SGRP9 ${ }^{P T o}$ NB confirms that $\mathrm{SGRP9}{ }^{P T o V}$ binding was specific. As observed in different ELISA experiments with OAc depleted sera, SGRP9 ${ }^{\text {PToV' }}$ 's binding to mammalian sera including rat and $\mathrm{Cmah}^{-/-}$mouse showed a linear and reproducible binding exclusive to base labile OAc esters (Suppl Fig S12H). The data showed that depletion of non-Oacetylated Sias doesn't influence SGRP9 ${ }^{P T o V}$ binding significantly while removal of OAc esters from Sias, or complete diminution of Sia population abolished its binding to sera (Suppl Fig 
$\mathrm{S} 12 \mathrm{H})$. These results together with sialoglycan microarray data (Fig1) confirm that SGRP9 ${ }^{\text {PToV }}$ exhibits very strong preferences towards 9OAcSias and does not interact with nonsialylated structures or proteins in complex biological samples.

\section{Western Blot analysis of SGRPs specificity towards sialoglycans in mammalian sera.}

To confirm that SGRPs are applicable to routine methods of glycoprotein analysis, we assessed SGRP's qualitative detection of sialoglycoproteins by western blots. The gel electrophoresis and Western blotting protocol was modified to minimize loss of sensitive OAc-Sias $(110,111)$. As observed, SGRP1 ${ }^{\text {YenB }}$ bound to all 9 sera included in the experiment, which agrees with the observation from serum binding ELISA experiments (Fig. 3A). SGRP2 ${ }^{\text {PltB }}$ also bound to sera in accordance with their Neu5Ac contents, with a noticeable difference between its affinity for WT and $\mathrm{Cmah}^{-/-}$mouse sera (Fig. 3C). SGRP3 ${ }^{H s a}$ and SGRP6 ${ }^{S N A}$ showed binding to all sera, a pattern that was also observed in serum binding ELISAs (Fig. 3E \& 3K). SGRP4 ${ }^{M H V}$ exclusively bound to guinea pig and horse sera (Fig. 3G), while SGRP9 ${ }^{P T o V}$ exhibited high binding towards rat, rabbit and $\mathrm{Cmah}^{-/-}$mouse sera (Fig. 3N), directly corresponding to OAc-Sia contents of these sera (Suppl Table 1). Interestingly, SGRP7 ${ }^{B C o V}$ showed significant binding with multiple sera which differs from our observations with sera binding ELISA experiments. In ELISA, SGRP7 ${ }^{B C O V}$ showed insignificant binding to sera and the overall binding pattern varied between experiments, suggesting a lack of linear binding behavior. We speculate that changes in $\mathrm{pH}$ and temperature during gel electrophoresis or blotting may result in migration of OAc esters, resulting in binding in western blotting, but we did not investigate this in detail. Blots, interrogated with non-binding variants of SGRPs (Fig. 3) did not show any binding to sera, suggesting Sia specific binding of SGRPs to sera. As SGRP8 ${ }^{T e T}$ did not bind sera in ELISA it was excluded from western blot analysis of serum proteins. 


\section{SGRP binding to frozen tissue sections: testing SGRP's utility through in situ detection of}

Sias. The panel shows the typical binding patterns observed with each of the probes (red color indicates binding) (Fig. 4). The non-binding control shows no binding to any of the sections, as expected. SGRP1 ${ }^{Y e n B}$ detects mucins and blood vessels in many organs, and this is best demonstrated in the sections of kidney, where the glomeruli are highlighted with this probe (Fig 4A). The glomeruli, as well as the capillary blood vessels between the tubules are prominent in the wild type mouse, while in the $\mathrm{Cmah}^{-/-}$mouse the capillaries between tubules are not as prominent (Fig 4B). The non-binding control shows no binding to any of the sections, as expected (Fig 4C). SGRP2 $2^{\text {PltB }}$ detects mucins and blood vessels in many organs, and this is best demonstrated in the sections of kidney, where the glomeruli are highlighted, and also the capillaries in between the tubules are visible (Fig 4D \& E). The non-binding control shows no binding to any of the sections, as expected (Fig 4F). SGRP3 ${ }^{H s a}$ detects mucins and blood vessels in many organs, and this is best demonstrated in the sections of kidney, where the glomeruli are prominently highlighted (Fig 4G). The binding to the kidney glomeruli in $\mathrm{Cmah}^{-/-}$mouse is slightly more prominent (Fig $4 \mathrm{H}$ ). The non-binding control shows no binding to any of the sections, as expected (Fig 4I). SGRP4 ${ }^{M H V}$ showed selective detection of mucins, and this is best demonstrated here in the mucin contained within the acini of pancreas. Staining was faint in the wild type and was much more prominently identified in the Cmah null animal (Fig 4J \&K). The islets of the pancreas (the endocrine portion) and the non-binding control show no evidence of binding (Fig 4L). SGRP5 ${ }^{\operatorname{Ig} Y}$ detects blood vessels in many organs and demonstrated here by the detection of the capillaries within the sections from the pancreas in wild type, but not in $\mathrm{Cmah}^{-/-}$mice (Fig 4M \&N). The non-binding control shows no binding to any of the sections (Fig 4O). SGRP6 ${ }^{S N A}$ detects mucins and blood vessels in many organs. Here we observe that SGRP6 ${ }^{S N A}$ detects blood vessels in the pancreas of the $\mathrm{Cmah}^{-/-}$mouse 
much better than it does in the wild type animal (Fig 4P \& Q). The tissue sections were incubated with secondary antibody Cy3-SA and served as control for SGRP6 ${ }^{S N A}$ specificity, and shows no binding to any of the sections, as expected (Fig 4R). SGRP7 ${ }^{B C o V}$ detects blood vessels in some organs but also detected white matter in the brain of the $C \mathrm{mah}^{-/}$mouse extremely well (Fig 4T). The non-binding control shows no binding to any of the sections. (Fig 4U). SGRP8 ${ }^{T e T}$ detected large areas of the non-nuclear neuropil in the brain from both the wild type and in the $\mathrm{Cmah}^{-/}$ mouse. The white matter in the brain from the $\mathrm{Cmah}^{-/}$mouse was even more prominent, and the non-binding control showed no binding to any of the sections (Fig 4V, W \& X). SGRP9 ${ }^{\text {ToV }}$ detected mucins and also red blood cells in many of the organs However, in sections of liver from the $\mathrm{Cmah}^{-/}$mouse, there was an abnormally high expression in the sinusoidal endothelial cells around the central vein, indicating right heart failure in the $\mathrm{Cmah}^{-/}$mouse (the portal triads, have two sources of blood supply and are thus the better perfused areas in the liver) (Fig 4Y \& Z). The non-binding control showed no binding to any of the sections, as expected (Fig 4Z1).

SGRP binding to mammalian erythrocytes in flow cytometry. We performed flow cytometry experiments with mammalian RBCs to demonstrate in situ detection of extracellular Sias by SGRPs (Fig. 5). As shown in Fig 5A, SGRP1 ${ }^{\text {YenB }}$ bound with RBCs from all 9 animal sources tested, while its nonbinding version SGRP $1^{\text {YenB }} \mathrm{NB}$ didn't show any binding (Fig 5A). In general, the total fluorescence intensity for nonbinder probes remained comparable to autofluorescence (RBCs without any treatment) and antibody control (RBCs detected with SA-PE only). SGRP2 $2^{P l t B}$ bound with all RBCs except wild type mouse. The most robust bindings of SGRP2 $2^{P l t B}$ were detected with $\mathrm{Cmah}^{-/}$mouse, guinea pig, rabbit and rat followed by goat and human RBCs (Fig 5B). SGRP2 $2^{P l t B} \mathrm{NB}$ didn't show any noticeable binding with RBCs from any source. SGRP3 ${ }^{H s a}$ bound with all types of RBCs with maximum binding detected from guinea pig, goat and rat RBCs 
and minimum with rabbit, indicating variable abundance of $\alpha 2-3$ Sias on these erythrocytes (Fig 5C). SGRP3 ${ }^{H s a} \mathrm{NB}$ didn't bind to any RBC type significantly. SGRP4 ${ }^{M H V}$ showed no noticeable binding to any RBC included in experiment, and the binding as detected from SGRP4 ${ }^{M H V}$ and SGRP $4{ }^{M H V}$ NB remained comparable, suggesting absence of 4 OAcSias in extracellular surface of these RBCs (Fig 5D). SGRP5 ${ }^{\operatorname{IgY}}$ bound with all RBCs except from $\mathrm{Cmah}^{-/}$mouse as anticipated (Fig 5E). Remarkably, SGRP5 ${ }^{\operatorname{Ig} Y}$ also didn't bind strongly to guinea pig and rat RBCs, suggesting less abundant Neu5Gc in these RBCs in comparison to mouse, horse and rabbit. SGRP5 ${ }^{\operatorname{Ig} Y} \mathrm{NB}$ showed negligible detection, as expected from a nonbinding control probe. A stronger SGRP6 ${ }^{S N A}$ binding was detected with human, guinea pig and rabbit in comparison to goat, sheep and horse (Fig 5F). Taking together with SGRP3 ${ }^{H s a}$ 's binding to RBCs, the relative abundance of $\alpha 2-3$ Sias over $\alpha 2-6$ Sias can be inferred for certain mammalian RBCs like goat, sheep and guinea pig. In absence of nonbinding mutant of SGRP $6^{S N A}$, the results are provided with autofluorescence and fluorescence antibody controls. The 7,9-diOAc-Sia-specific binder SGRP7 ${ }^{B C o V}$ showed no appreciable binding in flow cytometry experiment, suggesting undetectable amounts of its ligand in tested RBCs (Fig 5G). The resultant binding of SGRP7 ${ }^{B C o V}$ and $\mathrm{SGRP} 7^{B C O V} \mathrm{NB}$ remains comparable with RBCs; the results showed agreement with probe's response with serum ELISA experiment, suggesting lack of SGRP7 ${ }^{B C o V}$ binding in absence of its exclusive ligand 7,9-diOAcSia. $\alpha 2-8$-linked disialyl-oligosaccharides specific probe SGRP8 ${ }^{T e T}$ didn't show any binding with RBCs tested (Suppl Fig S13B) and hence a set of GD3 transfected CHOK1 cells were used to investigate efficiency of SGRP8 ${ }^{T e T}$ in flow cytometry experiment. As shown in Fig 5I, SGRP8 ${ }^{T e T}$ exhibited appreciable binding to GD3 expressing CHOK1 cells $\left(\mathrm{CHOK} 1-\mathrm{GD}^{+/+}\right)$with respect to normal CHOK1 cells. The nonbinding SGRP8 ${ }^{T e T} \mathrm{NB}$ showed relatively minor binding with cells, that was not affected by expression of GD3 gangliosides. As presented in Fig 5H, SGRP9 ${ }^{P T o V}$ 
showed remarkable binding with rat RBCs among all tested erythrocytes and its nonbinder probe SGRP9 ${ }^{P T o V} \mathrm{NB}$ remained completely undetectable throughout the experiment. This experiment demonstrates the utility of SGRPs in characterization of the extracellular sialome of cells -for example showing that rat RBCs possess a dense Sia population that contains varying amounts of in $\alpha 2-3-S i a s$, Neu5Ac and 9OAcSias9-OAc-Sias than $\alpha 2-6-S i a s$, Neu5Gc and 4OAc4-OAc-Sias.

\section{Conclusions and Perspectives}

In this study we exploited naturally evolved Sia-recognizing microbial proteins to develop a feasible approach to design and derive sialoglycan-detecting probes, and applied the approach to develop and characterize a set of practicable probes recognizing the most predominant classes of mammalian Sia types. The study also reviewed and re-examined current standing of traditional probes or probe-like molecules that have been reported against known diversity of mammalian Sia types. We reviewed, assessed, and experimentally analyzed several probes to compare their suitability for use as updated SGRPs. In this process, we successfully identified an alternative for Maackia lectins in the form of SGRP $3^{H s a}$. The report does underline the utility of conventional $\alpha 2-$ 6Sia-binding Sambucus nigra lectin which appeared to be better than several microbial and mammalian proteins tested or reviewed in this study. Among developed probes; SGRP2 $2^{\text {PltB }}$, SGRP4 ${ }^{M H V}$, SGRP8 ${ }^{T e T}$ and SGRP9 ${ }^{P T O V}$ represent novel probes for their class of Sia molecules, while SGRP1 ${ }^{\text {YenB }}$ and SGRP3 ${ }^{H s a}$ showed substantial improvement over previously known probes. SGRP5 $^{\operatorname{IgY} Y}$ was also included in the final set, and SGRP6 ${ }^{S N A}$ remained unique as we could not identify better alternative from microbial sources. Neu5Gc-specific microbial proteins showed utility but could not match the specificity and robustness of anti-Neu5Gc IgY, that was finally selected as SGRP5 ${ }^{\operatorname{Ig} Y}$. An exception to SGRPs set, $\mathrm{SGRP}^{B C o V}$ is characterized as an imperfect probe that is not completely selective for 7OAcSias, and that must be updated with more precise 
SGRP7 if one is identified in the future. Similarly, if a new probe is developed that is specific for Neu5Gc, that would replace the SGRP5 ${ }^{\operatorname{Ig} Y}$ which is currently selective for 7-OAc-Sias.

There are efforts ongoing by other groups, both in academic and industry to provide state of art sialoglycan detecting probes, and we attempted to review of most such studies and theoretically compared SGRPs with those molecules. Our focus remained on merits of proposed SGRPs and their utilities for a common biological science investigator. To have a consolidated assumption about the 'best' available probe for any given sialoglycan class, a real time comparison is required which is outside the scope of this study. In conclusion, this study provides a comprehensive set of probes for mammalian Sia types, designed and developed for the non-sialic acid experts. Several tests of demonstration of SGRPs specificity confirms their utility as practicable probes which can, and we expect SGRPs to be adapted by both experts and non-experts as tools of Sia recognition in their studies. 


\section{Experimental Procedures}

\section{Preparation of constructs:}

SGRP-1 - Y. enterocolitica Toxin B5; Y. enterocolitica Toxin B5 (Non-Binding): The full-length coding region of YESubB (423 bp) incorporating a 3' Hisx6 tag in the bacterial expression vector pBAD18 was kindly provided by James Paton and is described elsewhere. The corresponding nonbinding mutant YESubB (S31A; Y100F) was engineered from the wt template above using the Q5 Site-Directed Mutagenesis Kit (New England BioLabs) and sequence verified.

SGRP-2 - S. typhi toxin B5; S. typhi toxin B5 (Non-Binding): The Toxoid sequence pltB_pltA(E133A)_cdtB(H160Q)-6xHis and its corresponding non-binding control pltB(S35A)_pltA_cdtB-6xHis in the bacterial expression vector pET28b (Novagen) was kindly provided by Jorge Galan and described elsewhere. PltB or PltB(S35A) (411 bp) were amplified from the above respective constructs by PCR using pfu polymerase and primers 5, GCGCGCCATGGATGTATATGAGTAAGTATGTACC 3'; Nco1 site underlined and 5' ATATAAAGCTTCTTGGGTCCAAAGCATTGTGTCG; Hindlll site underlined. PCR products were digested with Ncol and Hindlll and sub-cloned into the corresponding sites of pET28b. Constructs were sequence verified.

SGRP-3 - S. gordonii hemagglutinin surface adhesion BR; S. gordonii hemagglutinin surface adhesion BR (Non-Binding): The HsaBR sequence and its corresponding non-binding control $\operatorname{Hsa}_{\mathrm{BR}}(\mathrm{R} 340 \mathrm{E})$ (709 bp) in the bacterial expression vector pGEX-3X (Millipore Sigma) was cloned by Paul Sullam's Laboratory and described elsewhere. Hsa $a_{\mathrm{BR}} / \mathrm{pGEX}-3 \mathrm{X}$ and $\operatorname{Hsa}_{\mathrm{BR}}(\mathrm{R} 340 \mathrm{E}) / \mathrm{pGEX}-3 \mathrm{X}$ were subsequently modified to incorporate a 3'SNAPf_Hisx6 tag. Briefly, a Hisx6 sequence tag was inserted into the BamH1 and Xho1 sites of MCS2 of pSNAPf (New England Biolabs) to create pSNAPf_Hisx6. SNAPf_6xHis was amplified from this construct 
by PCR using the forward primer 5'- AAAAGAATTCAACCATGGACAAAGACTGCGAAA-3' and reverse primer 5'- TTTTGAATTCATTAGTGATGGTGATGGTGATGGGA-3';

EcoR1 site underlined. PCR products were digested with EcoR1 and sub-cloned into the EcoR1 site of Hsa $\mathrm{BR}_{\mathrm{p}} \mathrm{pEEX}-3 \mathrm{X}$ and $\mathrm{Hsa}_{\mathrm{BR}}(\mathrm{R} 340 \mathrm{E}) / \mathrm{pGEX}-3 \mathrm{X}$ above. Constructs were sequence verified.

SGRP-4 - Mouse Hepatitis Virus-S Hemagglutinin Esterase (Inactive); Mouse Hepatitis VirusS Hemagglutinin Esterase (Non-Binding): The coding sequence of MHV-S Hemagglutinin Esterase inactive (S45A) (1218bp) fused to human IgG-Fc followed by a $6 x$ His tag in the mammalian expression vector pcDNA3.1(-) (Invitrogen) was kindly provided by Colin Parrish (MHV-S-HE_Fc_His6) and described elsewhere. The Hisx6 and associated stop codon was deleted by Q5 site directed mutagenesis (New England Biolabs). Two complimentary oligonucleotides corresponding to the Avi-tag (Avidity) sequence GGTCTGAACGACATCTTCGAGGCTCAGAAAATCGAATGGCACGAA incorporating Hindlll overlapping ends were synthesized (Integrated DNA Technologies) and annealed at $100^{\circ} \mathrm{C}$ for 5 mins followed by slow cooling. The annealed oligos were cloned into the Hindlll cut site of MHV-S-HE_Fc to produce MHV-S_HE_Fc_Avitag. Non-binding mutant (F212A) was generated from template MHV-S_HE_Fc_Avitag using the Q5 Site-Directed Mutagenesis Kit (New England BioLabs)

SGRP-7 - Bovine Coronavirus-Hemagglutinin Esterase (Inactive); Bovine Corona virusHemagglutinin Esterase (non-binding): The BCoV-Mebus HE ORF was amplified by PCR in junction overlap with a PCR amplification of the pFastBac1 clone of PToV-P4 He-Fc/Avi (see SGRP-9), but minus PToV HE sequence. Gibson assembly allowed for in-frame addition of BCoV-Mebus HE into the construct in direct replacement of PToV-P4 HE. This strategy 
maintained all vector, signal, and Fc-fusion/Avi architecture between constructs. Non-binding mutant F211A was generated by Q5 site-directed mutagenesis (New England Biolabs)

SGRP-8 - Clostridium tetani Heavy Chain/B Subunit; Clostridium tetani Heavy Chain/B Subunit (non-binding) mutant R1226L/W1289A; Clostridium tetani Heavy Chain/B Subunit (non-binding) mutant $\boldsymbol{W 1 2 8 9} \boldsymbol{A}$ - The optimal receptor-binding domain (OBD) (100, 100) of Tetanus Toxin (Heavy Chain/B Subunit) was synthesized by GENEWIZ (incorporating flanking 5' Hindlll and 3' Ncol restriction sites) and codon optimized for expression in E.Coli (a.a 1105>1315). pGEX-3X containing an irrelevant gene (IG) was engineered to contain a SNAPf_Hisx6 tag in the EcoR1 restriction site for use as an intermediate template (pGEX3X_IG_SNAPf_Hisx6) and described elsewhere. Briefly, a Hisx6 sequence tag was inserted into the BamH1 and Xho1 sites of MCS2 of pSNAPf(New England Biolabs) to create pSNAPf_Hisx6. SNAPf_6xHis was amplified from this construct by PCR using VENT Polymerase (NEB), forward primer 5'- AAAAGAATTCAACCATGGACAAAGACTGCGAAA-3' and reverse primer 5'TTTTGAATTCATTAGTGATGGTGATGGTGATGGGA-3'

(EcoR1 site are underlined). PCR products were digested with EcoR1 and sub-cloned into the EcoR1 site of the pGEX-3X_IG).pGEX-3X_IG_SNAPf_Hisx6 was then cut with Hindlll and Ncol to directly replace IG with Hindlll and Nco1 cut B Subunit (OBD). Non-binding double mutant R1226L/W1289A and non-binding single mutant W1289A were generated by Q5 sitedirected mutagenesis (New England Biolabs). Constructs were sequence verified.

\section{SGRP-9 - Porcine Torovirus Hemagglutinin Esterase (inactive);Porcine Torovirus}

Hemagglutinin Esterase (non-binding): The coding sequence of PToV Hemagglutinin Esterase inactive (S46A) and PToV Hemagglutinin Esterase non-binding (F271A) (1176bp) fused to human IgG-Fc followed by a $6 x$ His tag in the Insect expression vector pFastBac1 (ThermoFisher) was 
kindly provided by Colin Parrish (PToV-HE_S46A-Fc_Hisx6/pFastBac1; PToV-HE_F271A-

Fc_Hisx6/pFastBac1) and described elsewhere. Both PToV-HE_S46A-Fc_Hisx6 and PToVHE_F271A-Fc_Hisx6 were sub-cloned into pAC6 (Avidity) in-frame with the 3'Avitag (Avidity) sequence GGTCTGAACGACATCTTCGAGGCTCAGAAAATCGAATGGCACGAA. The resulting Avi-tagged constructs were amplified by PCR using pfu polymerase and the primers 5 ' ATATAGCGGCCGCTTTCTCGAGGCATAAGGAACACAC; Not1 site underlined and 3' CGCGCGGTACCTTATTCGTGCCATTCGATTTTCTGAG; Kpn1 site underlined. PCR products were digested with Not1 and Kpn1 and sub-cloned into the corresponding sites of PToVHE_S46A-Fc_Hisx6/pFastBac1 and PToV-HE_F271A-Fc_Hisx6/pFastBac1, which were cut with Not1 and Kpn1 thus removing the original untagged sequences. The final constructs were sequence verified.

Y.Pestis SubB - The full-length coding region of YPSubB (420 bp) incorporating a 3' Hisx6 tag in the bacterial expression vector pBAD18 was kindly provided by James Paton and is described elsewhere.

GspBBR; GSpBBR (non-binding); UB10712BR; UB10712BR (non-binding):: pGEX3XGspBBR $\triangle$ cnaA (containing just the Siglec and Unique domains of GspBBR) and pGEX3x10712BR were constructed previously (2016 Glycobiology). The SNAPf_Hisx6 coding sequence was inserted in-frame into the EcoRI site at the 3 'end of the BR sequences as described for SGRP3 above. The non-binding mutant BR- SNAPf_Hisx6 sequences were synthesized (LifeTechnologies) and then used to replace the BR segment (HindIII-NcoI) of pGEX3XGspBBR $\Delta$ cnaA-SNAPf_Hisx6.

E. coli SubB; E. coli SubB (Jennings mutant); E.coli SubB (non-binding): The coding region of E. coli SubB (423 bp) in pET-23 (+) bacterial expression vector was provided by Michael Jennings. 
Deletion of S106 and T107 was performed from the wt template above using the Q5 Site-Directed Mutagenesis Kit (New England BioLabs) and sequence verified. Mutant S106;T107 was cloned into pET28b which had been modified to contain a 3' SNAPf_Hisx6 tag. This was subsequently engineered to contain an S12A mutation (non-binding) using Q5 mutagenesis.

$\boldsymbol{C D 2 2 - F \boldsymbol { C }} \boldsymbol{A C P}$ - A CD22 cDNA fragment encoding the first two Ig-like domains fused to an EKhIgG-Fc fragment was amplified by PCR and cloned into the mammalian expression vector pACP$\operatorname{tag}(\mathrm{m})-2$ (New England Biolabs).

\section{Expression and purification of proteins in bacterial systems:}

Overexpression and purification of YenB and its nonbinding mutant: A single colony of BL21(DE3) competent cells (NEB, C2527H), transformed with YenB (or its mutant YenB $\mathrm{S} 31 \mathrm{~A} / \mathrm{Y} 100 \mathrm{~F}$ ) was inoculated in $25 \mathrm{ml}$ of LB media in presence of $100 \mu \mathrm{g} / \mathrm{ml}$ ampicillin (Millipore, 171254-25GM) and grown overnight at $37^{\circ} \mathrm{C}$ with mild shaking of $225 \mathrm{rpm} .20 \mathrm{ml}$ of this primary culture was added to 1 liter of LB media supplemented with $100 \mu \mathrm{g} / \mathrm{ml}$ ampicillin and grown at $37{ }^{\circ} \mathrm{C}$ until optical density at $600 \mathrm{~nm}$ reached to $0.2-0.3$. To induced protein overexpression, arabinose (Sigma, A3256-25G) was added to the culture to make final concentrations $0.2 \% \mathrm{v} / \mathrm{v}$, and the culture was incubated at $25^{\circ} \mathrm{C}$ for $5-6$ hours with mild shaking of $225 \mathrm{rpm}$. Bacteria was pelleted at $5000 \mathrm{~g}, 20 \mathrm{~min}, 4^{\circ} \mathrm{C}$ and suspended in resuspension buffer (50 mM Tris-HCl, pH 8, 300 mM NaCl, 2 mg/ml lysozyme (Sigma, L6876), 1 mM EDTA, 10\% glycerol in presence of DNAse (Sigma, D4263-5VL) and Protease inhibitors (Millipore, 5393134) at $4 \mathrm{~g} / \mathrm{ml}$. Pellet was resuspended, sonicated (30secs- ON/OFF, 10 cycles) and centrifuged (10000 $\left.\mathrm{g}, 4{ }^{\circ} \mathrm{C}, 30 \mathrm{~min}\right)$ to exclude insoluble fractions. The soluble fraction was resuspended with preequilibrated Ni-NTA resin (Qiagen, 1018600) and incubated for $1 \mathrm{hr}$ at $4{ }^{\circ} \mathrm{C}$ with end-to-end 
rotation. This slurry was passed through purification column (Biorad, PolyPrep 731-1550) and washed with $5 \mathrm{ml}$ of $50 \mathrm{mM}$ Tris- $\mathrm{HCl}, 300 \mathrm{mM} \mathrm{NaCl}$, pH 8, followed by 2 washes with $5 \mathrm{ml}$ of $50 \mathrm{mM}$ Tris-HCl, $300 \mathrm{mM} \mathrm{NaCl}$ pH 8, 30 mM Imidazole (Sigma, I2399-100G). The protein was eluted in 7 aliquots of $700 \mu \mathrm{l}$ each with elution buffer $(50 \mathrm{mM}$ Tris- $\mathrm{HCl}, 100 \mathrm{mM} \mathrm{NaCl}, 300 \mathrm{mM}$ Imidazole supplemented with protease inhibitors) and analyzed by SDS-PAGE. Aliquots with optimal protein concentrations were collected and transferred to PBS (Millipore, Amicon ultra centrifuge filter), supplemented with protease inhibitors, quantified (BCA, Pierce, Thermo Scientific 23225 ) and saved at $-80^{\circ} \mathrm{C}$ until further steps.

Overexpression and purification of PltB and its nonbinding mutant: A single colony of BL21DE competent cells (NEB, C2527H), transformed with PltB (or its mutant- PltB S35A) was inoculated in $25 \mathrm{ml}$ of LB media in presence of $50 \mu \mathrm{g} / \mathrm{ml}$ kanamycin (Sigma, K1377-5G) and grown overnight at $37{ }^{\circ} \mathrm{C}$ with mild shaking of $225 \mathrm{rpm} .10 \mathrm{ml}$ of this primary culture was added to 0.5 lit of LB media (Millipire, 1.10285 .0500 ) supplemented with $50 \mu \mathrm{g} / \mathrm{ml}$ kanamycin and grown at $37^{\circ} \mathrm{C}$ until optical density at $600 \mathrm{~nm}$ reached to $0.6-0.7$. PltB overexpression was induced by $0.5 \mathrm{mM}$ IPTG (Apex, 20-109) at $29^{\circ} \mathrm{C}$ for $12-14$ hours with shaking of $250 \mathrm{rpm}$. Bacteria was pelleted at 5000 g, $20 \mathrm{~min}, 4{ }^{\circ} \mathrm{C}$ and suspended in resuspension buffer $(50 \mathrm{mM}$ Tris- $\mathrm{HCl}, \mathrm{pH} \mathrm{8,} 150 \mathrm{mM} \mathrm{NaCl}, 2$ mg/ml lysozyme, $1 \mathrm{mM}$ EDTA, DNAse and protease inhibitors) at $4 \mathrm{~g} / \mathrm{ml}$. Pellet was resuspended, sonicated (30 secs ON/OFF, 10 cycles, Fischer Scientific, 550 Sonic Dismembrator) and centrifuged (10000 g, $4{ }^{\circ} \mathrm{C}, 30 \mathrm{~min}$ ) to exclude insoluble fractions (Sorvall, RC6 plus, Thermo). The soluble fraction was resuspended with preequilibrated Ni-NTA resin (Qiagen, 1018600) and incubated for $1 \mathrm{hr}$ at $4{ }^{\circ} \mathrm{C}$ with end to end rotation. This slurry was passed through purification column (Biorad, PolyPrep 731-1550) and washed with $5 \mathrm{ml}$ of $50 \mathrm{mM}$ Tris-HCl, $150 \mathrm{mM} \mathrm{NaCl}$, $\mathrm{pH}$, followed by 2 washes with $5 \mathrm{ml}$ of $50 \mathrm{mM}$ Tris-HCl, $300 \mathrm{mM} \mathrm{NaCl}, \mathrm{pH} 8,50 \mathrm{mM}$ Imidazole 
(Sigma, I2399-100G). The protein was eluted in 7 aliquots of $700 \mu 1$ each with elution buffer (50 $\mathrm{mM}$ Tris-HCl, $100 \mathrm{mM} \mathrm{NaCl}, 300 \mathrm{mM}$ Imidazole supplemented with protease inhibitors) and analyzed by SDS-PAGE. Aliquots with optimal protein concentrations were collected and transferred to PBS, supplemented with protease inhibitors, quantified (BCA, Pierce, Thermo Scientific 23225) and saved at $-80{ }^{\circ} \mathrm{C}$ until further steps.

Overexpression and purification of $\mathrm{Hs}_{B R}$ and its nonbinding mutant: A single colony of BL21(DE3) competent cells, transformed with HsaBR (or its mutant HsaBR R340E) was inoculated in $25 \mathrm{ml}$ of LB media in presence of $100 \mu \mathrm{g} / \mathrm{ml}$ ampicillin (Millipore, 171254-25GM) and grown overnight at $37^{\circ} \mathrm{C}$ with mild shaking of $225 \mathrm{rpm} .10 \mathrm{ml}$ of this primary culture was added to 0.5 lit of LB media supplemented with $100 \mu \mathrm{g} / \mathrm{ml}$ ampicillin and grown at $37{ }^{\circ} \mathrm{C}$ until optical density at $600 \mathrm{~nm}$ reached to $0.7-0.8$. Protein overexpression was induced by $0.5 \mathrm{mM}$ IPTG (Apex, 20-109) at $24{ }^{\circ} \mathrm{C}$ for $12-14$ hours (or $37^{\circ} \mathrm{C}, 3 \mathrm{hrs}$ ) with shaking of $250 \mathrm{rpm}$. Bacteria was pelleted at 5000 $\mathrm{g}, 20 \mathrm{~min}, 4^{\circ} \mathrm{C}$ and suspended in resuspension buffer (50 mM Tris- $\mathrm{HCl}, \mathrm{pH}$ 7.5, $150 \mathrm{mM} \mathrm{NaCl}, 2$ $\mathrm{mg} / \mathrm{ml}$ lysozyme, $1 \%$ Triton X-100, $1 \mathrm{mM}$ EDTA, DNAse and protease inhibitors) at $4 \mathrm{~g} / \mathrm{ml}$. Pellet was resuspended, sonicated ( $30 \mathrm{secs} \mathrm{ON} / \mathrm{OFF}, 8$ cycles) and centrifuged (10000 g, $\left.4{ }^{\circ} \mathrm{C}, 30 \mathrm{~min}\right)$ to exclude insoluble fractions. The soluble fraction was resuspended with preequilibrated GST Sepharose resin (Thermo, 17-0756-01) and incubated for $1 \mathrm{hr}$ at $4{ }^{\circ} \mathrm{C}$ with end to end rotation. This slurry was passed through purification column (Biorad, PolyPrep 731-1550) and washed with 10 bed volumes of resin by $50 \mathrm{mM}$ Tris- $\mathrm{HCl}, \mathrm{pH}$ 7.5, $150 \mathrm{mM} \mathrm{NaCl}, 1 \mathrm{mM}$ PMSF, 0.5\% Triton $\mathrm{X}-100$ twice, followed by 2 washes with 10-bed-volume with $50 \mathrm{mM}$ Tris-HCl, pH 7.5, $150 \mathrm{mM}$ $\mathrm{NaCl}, 1 \mathrm{mM}$ PMSF. The protein was eluted in 7 aliquots of $700 \mu \mathrm{l}$ each with elution buffer (10 $\mathrm{mM}$ reduced glutathione (Sigma 4251-5G in $50 \mathrm{mM}$ Tris-HCl, $\mathrm{pH}$ 8, $150 \mathrm{mM} \mathrm{NaCl}$, supplemented with protease inhibitors) and analyzed by SDS-PAGE. Aliquots with optimal protein 
concentrations were collected and transferred to PBS, supplemented with protease inhibitors, quantified by BCA and saved at $-80{ }^{\circ} \mathrm{C}$ until further steps. Similar procedures were followed for expression and purification of $\mathrm{GspB}_{\mathrm{BR}}, \mathrm{UB} 10712_{\mathrm{BR}}$ and their nonbinding mutants.

Overexpression and purification of TeT-HCR, TeT-HCR 1226L, TeT-HCR W1289A and its nonbinding mutant: The procedure to purify TeT-HCR and its variant proteins remained comparable to protocol adopted for HsaBR, except for the IPTG induction conditions. For TeT$\mathrm{HCR}$, proteins were overexpressed at $26^{\circ} \mathrm{C}$ for $4 \mathrm{hr}$ with shaking at $250 \mathrm{rpm}$.

\section{Expressions and purification of proteins in mammalian expression system:}

\section{Overexpression and purification of $M H V-H E-F c$ and $h C D 22$ Fc:}

HEK293 cells were transiently transfected by endotoxin free construct of MHV-Fc virolectin or its mutant in presence of polyethyleneimine (PEI, Polyscience Inc, 23966). Precisely, $18 \mu \mathrm{g}$ DNA was vortexed with $54 \mu \mathrm{l}$ of $1 \mathrm{mg} / \mathrm{ml}$ PEI in $2.4 \mathrm{ml}$ of advanced DMEM (no serum, Gibco-12491015 ) and added to each of $15 \mathrm{~cm}$ dish of $80-90 \%$ confluent cell in $20 \mathrm{ml}$ (advanced DMEM, $2 \% \mathrm{FCS}, 1 \mathrm{X} \mathrm{P} / \mathrm{S}, 1 \mathrm{X}$ glutamine) and incubated $24 \mathrm{hr}$ at $37{ }^{\circ} \mathrm{C}$ in incubator. Post incubation, the media was replaced by $20 \mathrm{ml}$ basal media (96 ml RPMI 1640 (Invitrogen 11875-135), $96 \mathrm{ml}$ DMEM (Invitrogen 12430-104), $2 \mathrm{ml}$ of antibiotics 100X, $2 \mathrm{ml} 200 \mathrm{mM}$ L-glutamine, $2 \mathrm{ml} 100 \times$ Nutridoma (Nalgene, Nutridoma-SP, 11011375001), $2 \mathrm{ml}$ of $100 \mathrm{mM}$ sodium pyruvate) per plate and incubated for 4 days. The media was gently collected and centrifuged at $1000 \mathrm{~g}, 15 \mathrm{~min}, 4{ }^{\circ} \mathrm{C}$ to remove cell debris. The supernatant was adjusted to $10 \mathrm{mM}$ Tris-HCl, $\mathrm{pH}$ 8, filtered with a 0.2 $\mu \mathrm{M}$ filter unit (Corning, 430320) and incubated with $0.5 \mathrm{ml}$ protein-A-sepharose (nProtein A Sepharose 4 Fast flow, GE healthcare \#1705280) at $4{ }^{\circ} \mathrm{C}$ for $24 \mathrm{hr}$. Resin was collected in

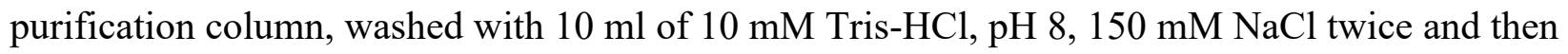


resin was resuspended in $2 \mathrm{ml}$ of $20 \mathrm{mM}$ HEPES $\mathrm{pH} 7$. Resin was treated with $25 \mathrm{mU}$ of Arthrobacter ureafaciens sialidase (EY laboratory, EC-32118-5) at room temperature for $1 \mathrm{hr}$ and then washed with $10 \mathrm{ml}$ of $10 \mathrm{mM}$ Tris-HCl, $\mathrm{pH} 8,150 \mathrm{mM} \mathrm{NaCl}$ to remove sialidase. MHV-Fc protein was eluted with $2 \mathrm{ml}$ of $0.1 \mathrm{M}$ glycine $\mathrm{pH} 3.0$ in a tube, already containing $0.3 \mathrm{ml}$ of cold $1 \mathrm{M}$ Tris-HCl, $\mathrm{pH} 8$, to neutralize $\mathrm{pH}$. The elutant was buffer exchanged to PBS, supplemented with protease inhibitors, quantified by BCA assay and saved at $-80^{\circ} \mathrm{C}$ until further steps. Similar procedure was followed for expression and purification of hCD22-Fc.

\section{Expressions and purification of proteins in insect expression system:}

Clones of pFastBac1 constructs were used to generate recombinant bacmids in DH10Bac following the manufacturers protocol (ThermoFisher). Recombinant baculoviruses were recovered by transfection of the bacmids into Sf9 insect cells using TransIt-Insect reagent (Mirus). Viruses were then used to infect suspension High Five cells grown in Express5 media and supernatants harvested $\sim 60-72$ hours post-infection. Proteins were purified from the infected cell culture supernatants by binding to a HiTrap ProteinG HP column (GE Healthcare Life Sciences, Piscataway, NJ) and eluted with $0.1 \mathrm{M}$ citrate, $\mathrm{pH} 3.0$ (pH neutralization to 7.8 with $1 \mathrm{M}$ Tris-HCl, pH 9.0) using ÄKTA FPLC system (GE Healthcare Life Sciences). The HE-Fc containing fractions were dialyzed in PBS and concentrated using 30kD Amicon Ultra-15 filters (EMD Millipore). Purified proteins were observed by SDS gel migration as well as anti-human IgG Fc probing by Western. Protein concentrations were determined from A280 readings and MicroBCA Assay. Proteins were primarily aliquoted and stored at $-80^{\circ} \mathrm{C}$.

Biotinylation of Proteins: Biotinylation of SNAP tagged proteins and Avi-tagged proteins were performed as suggested by manufactures (NEB, Avidity). Direct biotinylation of proteins for example-YenB, PltB and Neu5Gc-IgY was achieved by NHS mediated biotin tagging at 20:1 
molar ratio as per manufacturer's protocol (Thermo scientific, EZ-Link Sulfo-NHS-LC Biotin, Cat\#21326). Biotinylated proteins were concentrated, and buffer exchanged to PBS with microcon centrifugation columns according to the molecular weights of proteins. Finally, elutions were estimated for protein concentration and stored in $-80^{\circ} \mathrm{C}$ freezer. In addition to the functional assays on sialoglycan microarray and sera ELISA experiments, high efficiency of biotinylation was also confirmed by a pull-down assay using streptavidin beads (Thermo, 20347). Briefly, proteins biotinylated and mock biotinylated were tested for their affinity and subsequently elution from streptavidin beads. Pulled down biotinylated proteins were also compared parallelly to the initial concentration of protein to observe the approximate fractions of proteins acquiring biotin in biotinylation tagging procedures.

Sialoglycan microarray with SGRPs: As described by accompanying article by Sasmal et al.

Sialic acid release, DMB derivatization, and HPLC analysis: Bound Sias from serum samples were released using $2 \mathrm{M}$ acetic acid hydrolysis, $80{ }^{\circ} \mathrm{C}$ for 3 hour. The hydrolyzed samples were passed through a Microcon-10 filter (Sigma-Millipore); the released Sias were recovered in the filtrate. The Sias were derivatized with DMB (112). Briefly, 7 mM DMB, 1.4 M acetic acid, 0.75 $\mathrm{M} \beta$-mercaptoethanol, $18 \mathrm{mM}$ sodium hydrosulfite, at $50^{\circ} \mathrm{C}$ for $2.5 \mathrm{hour}$, in the dark. The DMBSias were separated on a Gemini C18 column, $4.6 \times 250 \mathrm{~mm}, 5 \mu$ (Phenomenex; Torrance, CA). Isocratic elution was employed using 7\% methanol, $8 \%$ acetonitrile, $85 \%$ water for 50 min at 0.9 $\mathrm{ml} / \mathrm{min}$ flow. The eluant was monitored by fluorescence with Ex373 and Em448. Sialic acid quantitation was done by derivatizing commercial Neu5Ac (Nacalai, USA; San Diego, CA) and comparing the area under the peak of known amounts.

Serum Binding assay with SGRPs: A saturable amount of sera (equivalent to $50 \mu \mathrm{g}$ proteins), reconstituted in PBST (0.1\% Tween 20) were added to individual wells in ELISA plates (Corning, 
9018) and kept overnight at $4^{\circ} \mathrm{C}$ for adherence. Unattached sera were removed, and wells were briefly rinsed with PBST before addition of $200 \mu 1,0.5 \%$ cold fish gelatin (Sigma- G7765-1L, diluted in PBST) for blocking. Lectins, SGRPs or other probes were reconstituted in diluting buffer (PBST with $0.05 \%$ cold fish gelatin) at desired concentrations and added to serum coated wells for $1 \mathrm{hr}$ (or otherwise specified) at room temperature. Specifically, for MAL-I (VECTOR LAB, B1315) and MAL-II (B1265), $10 \mathrm{mM} \mathrm{CaCl}_{2}$ and $10 \mathrm{mM} \mathrm{MnCl}_{2}$ were added to diluting buffer to maximize lectin binding. Post incubation, wells were vigorously washed with $250 \mu 1 \mathrm{PBST}, 5$ times and then incubated with Avidin-HRP (Biolegend, 405103) for biotinylated probes at 1:2000 dilution for 45 minutes at room temperature. Wells were again washed vigorously washed with $250 \mu \mathrm{l}$ PBST for 6-7 times. $100 \mu \mathrm{l}$ of TMB (BD OptEIA-55214) was added to each well as substrate for HRP's enzymatic activities and incubated for color development. The reactions were stopped by adding $40 \mu \mathrm{l}$ of $2 \mathrm{~N} \mathrm{H}_{2} \mathrm{SO}_{4}$ after 30 minutes, or if the wells specified as negative controls begin to develop color. Plates were read at $450 \mathrm{~nm}$ and readings were processed to observe binding patterns of tested molecules (Perkin Elmer, Enspire Alpha). For every experiment, wells treated with avidin-HRP only served as negative control of binding, along with non-binding mutants of probes, whenever applicable.

For serum binding experiment with nonbiotinylated proteins, binding of Fc-tagged proteins was probed by whereas binding of His $_{6}$-conjugated proteins was probed using anti-His antibody (mouse, Gene Script, \#A00186, 1:4000) and subsequently with anti-mouse-HRP (CTS, \#7076S, 1:1500). In certain probes discussed in article (for example, LFA (EY Lab, BA1501-1, CHE-FcD etc.), the pre-conjugated combination of probe and secondary antibody was adopted for stronger signals, as mentioned in corresponding figure legends. 
Mild Periodate and Base treatment of sera: To remove $O$-acetyl esters and render all Sias sensitive to mild periodate treatment, serum-coated ELISA plates were expose to strong $\mathrm{NH}_{4} \mathrm{OH}$ vapors for $24 \mathrm{hrs}$ at room temperature. In parallel, another plate coated with sera were incubated in humidified chamber filled with triple distilled water at room temperature as control set of base treated sera. Wells were washed 3 times with PBS briefly to remove any residual $\mathrm{NH}_{4} \mathrm{Cl}$. Subsequently, plates were washed three times with PBS $\mathrm{pH} 6.5$ to pre-equilibrate for mild periodate treatment, if required. To ascribe Sia specificity, serum-coated ELISA plates were treated with mild periodate to selectively cleave the Sia side- chain. For sodium periodate treatment in base treated/untreated plate, wells were incubated with $200 \mu 1$ of freshly prepared cold $2 \mathrm{mM}$ sodium metaperiodate (Fischer, S398-100) in PBS, pH 6.5 in dark, $4^{\circ} \mathrm{C}$, precisely for $20 \mathrm{~min}$. The reaction was stopped by the addition of $50 \mu \mathrm{l}$ of $100 \mathrm{mM}$ sodium borohydride (Fischer, S678-25) in PBS, $\mathrm{pH} 6.5$ (final concentration of $20 \mathrm{mM}$ ), followed by a 10 -min incubation at room temperature with gentle shaking (the borohydride inactivates the periodate). Concurrently, as a mock control, periodate and borohydride solutions were premixed (4:1), and wells were incubated with $250 \mu \mathrm{l} /$ well side by side with the periodate-treated wells. To remove resulting borates, wells were then washed three times (10 min each wash) with $50 \mathrm{mM}$ sodium acetate, $\mathrm{pH}$ 5.5, containing $100 \mathrm{mM} \mathrm{NaCl}$, followed by three washes with PBS, $\mathrm{pH}$ 7.4. Base and/or mild periodate treated sera were used to determine Sia specific serum binding, or OAc ester specific Sia binding in SGRPs or lectins. (113)

Western Blotting. Commercial animal sera (rat, equine, goat, guinea pig, rabbit, sheep; Sigma, St.Louis, MO), and wt mouse and human serum (Valley Biomedical, Winchester, VA) and in house $\mathrm{Cmah}^{-/}$mouse serum were chromatographed on 12\% CosmoPAGE Bis-Tris precast gels (Nacalai, USA, NU00212). The gels were run in CosmoPAGE MOPS running buffer pH 7 
(Nacalai, USA, NU01004), with ice packs to keep running buffer temperature below $23{ }^{\circ} \mathrm{C}$. The proteins were transferred to Immobilon FL (EMD Millipore Corp IPFL00010) membrane in transfer buffer containing 10\% methanol and 0.2\% SDS. Membranes were blocked overnight at 4 ${ }^{\circ} \mathrm{C}$ with $0.5 \%$ Cold water fish skin gelatin in PBST (PBS pH 7.4 with $0.1 \%$ Tween 20), with gentle agitation. The membranes were incubated at room temperature for $2 \mathrm{hrs}$ with $1 \mu \mathrm{g} / \mathrm{ml}$ with all but SGRP5 ${ }^{\operatorname{Ig} Y}$, which was used at $0.33 \mu \mathrm{g} / \mathrm{ml}$, diluted with blocking buffer without Tween. Also, SGRP4 ${ }^{M H V}$ and SGRP9 ${ }^{P T o V}$ were used at $2 \mu \mathrm{g} / \mathrm{ml}$. Amounts of serum proteins immunoblotted for each SGRP were adjusted to highlight their ligand preferences. Specifically, $25 \mu \mathrm{g}$ protein per well was loaded for SGRP1 ${ }^{\text {YenB }}$, SGRP2 $2^{P l t B}$ and their nonbinding variants, $50 \mu \mathrm{g}$ protein per well was loaded for SGRP3 ${ }^{H s a}$, SGRP4 ${ }^{M H V}$, SGRP7 ${ }^{B C o V}$, SGRP9 ${ }^{P T o V}$ and their nonbinding mutants, while $12.5 \mu \mathrm{g}$ protein per well was loaded for SGRP5 ${ }^{\operatorname{Ig} Y}$, SGRP5 ${ }^{\operatorname{Ig} Y} \mathrm{NB}$ and SGRP6 ${ }^{S N A}$ (Vector lab, B1305). Secondary antibody incubation was with Streptavidin IRDye 680 (Licor, 925-68079) at 1:10,000 dilution in PBST at room temperature. Signal was read with Odyssey infrared imager (Licor Biosciences, Lincoln, NE). Interrogating SGRPs and corresponding non-binding SGRPs were used at the same concentration and all images were obtained at the same time using the same imager settings.

Histology Procedure: Frozen sections of various organs from the wild type and $\mathrm{Cmah}^{-/-}$mouse were air dried for 30 minutes, rehydrated in Tris-Buffered Saline $\mathrm{pH} 7.5$ and overlaid with $0.5 \%$ Fish gelatin, which was then tipped off for blocking the endogenous biotin suing the Avidin Biotin blocking kit from Vector labs (SP-2001). Sections were then fixed for 30 minutes with 10\% Neutral buffered formalin (VWR-89370-094), washed and overlaid with either the binding or nonbinding probes, each used 5 micrograms/milliliter. Separate slides (Globe, 1358W) were then overlaid with each of the Biotinylated probes and incubated in a humid chamber at room 
temperature for 30 minutes, followed by washing in TBS and then overlaid with Cy3 Streptavidin (Red, Jackson Laboratory, 016-160-084) for 30 minutes, washed and then nuclei were counterstained with Hoechst (Blue, Molecular Probes, H3570) for two minutes, before washing and cover-slipping using an aqueous mounting medium (Vector lab H5501). Digital images were captured using the Keyence microscope, and organized using Adobe Photoshop.

Flow Cytometry: SGRPs and probes were probed at $5 \mu \mathrm{g} / \mathrm{ml}$ (or otherwise, mentioned in figure legends) for their RBC binding properties. For this experiment, blood from several animal species was resuspended in PBS $\left(4{ }^{\circ} \mathrm{C}\right)$ to make $2 \%$ volume/volume dilution. $200 \mu$ of this blood suspension was supplemented with $200 \mu \mathrm{l}$ of $10 \mu \mathrm{g} / \mathrm{ml}$ probe dilution in PBS $\left(4^{\circ} \mathrm{C}\right)$ in a tube to make final blood suspension and probe dilutions at $1 \%$ and $5 \mu \mathrm{g} / \mathrm{ml}$ (unless mentioned otherwise). $400 \mu \mathrm{l}$ of this blood suspension was incubated ice for $1 \mathrm{hr}$ with intermittent gentle mixing to avoid settlement of RBCs. After incubation, $1 \mathrm{ml}$ ice cold PBS was added to each tube and centrifuged at 1000 RPM, 3 minutes at $4{ }^{\circ} \mathrm{C}$. The washing was repeated 2 more times, and then pellet was gently broken into $300 \mu \mathrm{l}$ of $1 \mu \mathrm{g} / \mathrm{ml}$ dilution of Streptavidin Cy3 (Jackson 016-170-084) and incubated for 45minutes in ice. For autofluorescence control of RBCs, blood suspension was never treated either by probe or fluorescence antibody, while for fluorescent secondary antibody control, a mock treated blood was incubated with equivalent streptavidin-Cy3 dilution in similar conditions. After incubations, all tubes were added with $1 \mathrm{ml}$ ice cold PBS and washed 3 times with $1 \mathrm{ml}$ PBS. Final pellet was gently broken in $500 \mu \mathrm{l}$ of PBS and fluorescence was analyzed in FL2 channel in FACS Caliber flow cytometer. The settings for acquisition of data and analysis remained same for all probes and their nonbinding mutants in an experiment. 
bioRxiv preprint doi: https://doi.org/10.1101/2021.05.28.446202; this version posted May 28, 2021. The copyright holder for this preprint (which

was not certified by peer review) is the author/funder, who has granted bioRxiv a license to display the preprint in perpetuity. It is made available under aCC-BY-NC-ND 4.0 International license.

\section{Acknowledgments}

This work was supported by NIH Grants U01CA199792 and R01GM32373 (to A.V.), and additional generous financial support from BioLegend, San Diego.

\section{Conflicts of Interest}

The authors declare no competing interests. 


\section{References}

1. Varki, A. and Kornfeld, S. (2015) in Essentials of Glycobiology (Varki, A., Cummings, R. D., Esko, J. D., Stanley, P., Hart, G. W., Aebi, M., Darvill, A. G., Kinoshita, T., Packer, N. H., Prestegard, J. H., Schnaar, R. L. and Seeberger, P. H., eds.) Cold Spring Harbor Laboratory Press, Cold Spring Harbor (NY)

2. Chen, X. and Varki, A. (2010) Advances in the biology and chemistry of sialic acids. ACS Chem Biol 5, 163-176

3. Schauer, R. and Kamerling, J. P. (2018) Exploration of the Sialic Acid World. Adv Carbohydr Chem Biochem 75, 1-213

4. Severi, E., Hood, D. W. and Thomas, G. H. (2007) Sialic acid utilization by bacterial pathogens. Microbiology 153, 2817-2822

5. Matrosovich, M., Herrler, G. and Klenk, H. D. (2015) Sialic Acid Receptors of Viruses. Top Curr Chem 367, 1-28

6. Kawanishi, K., Saha, S., Diaz, S., Vaill, M., Sasmal, A., Siddiqui, S. S., Choudhury, B., Sharma, K., Chen, X., Schoenhofen, I. C., Sato, C., Kitajima, K., Freeze, H. H., MünsterKühnel, A. and Varki, A. (2021) Evolutionary conservation of human ketodeoxynonulosonic acid production is independent of sialoglycan biosynthesis. J Clin Invest 131, 137681

7. Cummings, R. D., Darvill, A. G., Etzler, M. E. and Hahn, M. G. (2015) in Essentials of Glycobiology (Varki, A., Cummings, R. D., Esko, J. D., Stanley, P., Hart, G. W., Aebi, M., Darvill, A. G., Kinoshita, T., Packer, N. H., Prestegard, J. H., Schnaar, R. L. and Seeberger, P. H., eds.) Cold Spring Harbor Laboratory Press, Cold Spring Harbor (NY)

8. Bhavanandan, V. P. and Katlic, A. W. (1979) The interaction of wheat germ agglutinin with sialoglycoproteins. The role of sialic acid. J Biol Chem 254, 4000-4008

9. Miller, R. L., Collawn, J. F. and Fish, W. W. (1982) Purification and macromolecular properties of a sialic acid-specific lectin from the slug Limax flavus. J Biol Chem 257, 75747580

10. Byres, E., Paton, A. W., Paton, J. C., Löfling, J. C., Smith, D. F., Wilce, M. C., Talbot, U. M., Chong, D. C., Yu, H., Huang, S., Chen, X., Varki, N. M., Varki, A., Rossjohn, J. and Beddoe, T. (2008) Incorporation of a non-human glycan mediates human susceptibility to a bacterial toxin. Nature 456, 648-652

11. Deng, L., Song, J., Gao, X., Wang, J., Yu, H., Chen, X., Varki, N., Naito-Matsui, Y., Galán, J. E. and Varki, A. (2014) Host adaptation of a bacterial toxin from the human pathogen Salmonella Typhi. Cell 159, 1290-1299

12. Nguyen, T., Lee, S., Yang, Y. A., Ahn, C., Sim, J. H., Kei, T. G., Barnard, K. N., Yu, H., Millano, S. K., Chen, X., Parrish, C. R. and Song, J. (2020) The role of 9-O-acetylated glycan receptor moieties in the typhoid toxin binding and intoxication. PLoS Pathog 16, e1008336

13. Kawaguchi, T., Matsumoto, I. and Osawa, T. (1974) Studies on hemagglutinins from Maackia amurensis seeds. J Biol Chem 249, 2786-2792

14. Knibbs, R. N., Goldstein, I. J., Ratcliffe, R. M. and Shibuya, N. (1991) Characterization of the carbohydrate binding specificity of the leukoagglutinating lectin from Maackia amurensis. Comparison with other sialic acid-specific lectins. J Biol Chem 266, 83-88

15. Brinkman-Van der Linden, E. C., Sonnenburg, J. L. and Varki, A. (2002) Effects of sialic acid substitutions on recognition by Sambucus nigra agglutinin and Maackia amurensis hemagglutinin. Anal Biochem 303, 98-104 
16. Konami, Y., Yamamoto, K., Osawa, T. and Irimura, T. (1994) Strong affinity of Maackia amurensis hemagglutinin (MAH) for sialic acid-containing Ser/Thr-linked carbohydrate chains of N-terminal octapeptides from human glycophorin A. FEBS Lett 342, 334-338

17. Bai, X., Brown, J. R., Varki, A. and Esko, J. D. (2001) Enhanced 3-O-sulfation of galactose in Asn-linked glycans and Maackia amurensis lectin binding in a new Chinese hamster ovary cell line. Glycobiology 11, 621-632

18. Kaku, H., Mori, Y., Goldstein, I. J. and Shibuya, N. (1993) Monomeric, monovalent derivative of Maackia amurensis leukoagglutinin. Preparation and application to the study of cell surface glycoconjugates by flow cytometry. J Biol Chem 268, 13237-13241

19. Bensing, B. A., Khedri, Z., Deng, L., Yu, H., Prakobphol, A., Fisher, S. J., Chen, X., Iverson, T. M., Varki, A. and Sullam, P. M. (2016) Novel aspects of sialoglycan recognition by the Siglec-like domains of streptococcal SRR glycoproteins. Glycobiology 26, 1222-1234

20. Bensing, B. A., Lopez, J. A. and Sullam, P. M. (2004) The Streptococcus gordonii surface proteins GspB and Hsa mediate binding to sialylated carbohydrate epitopes on the platelet membrane glycoprotein Ibalpha. Infect Immun 72, 6528-6537

21. Deng, L., Bensing, B. A., Thamadilok, S., Yu, H., Lau, K., Chen, X., Ruhl, S., Sullam, P. M. and Varki, A. (2014) Oral streptococci utilize a Siglec-like domain of serine-rich repeat adhesins to preferentially target platelet sialoglycans in human blood. PLoS Pathog 10, e1004540

22. Takamatsu, D., Bensing, B. A., Cheng, H., Jarvis, G. A., Siboo, I. R., Lopez, J. A., Griffiss, J. M. and Sullam, P. M. (2005) Binding of the Streptococcus gordonii surface glycoproteins GspB and Hsa to specific carbohydrate structures on platelet membrane glycoprotein Ibalpha. Mol Microbiol 58, 380-392

23. Kamerling, J. P. and Gerwig, G. J. (2006) Structural analysis of naturally occurring sialic acids. Methods Mol Biol 347, 69-91

24. Klein, A. and Roussel, P. (1998) O-acetylation of sialic acids. Biochimie 80, 49-57

25. Cariappa, A., Takematsu, H., Liu, H., Diaz, S., Haider, K., Boboila, C., Kalloo, G., Connole, M., Shi, H. N., Varki, N., Varki, A. and Pillai, S. (2009) B cell antigen receptor signal strength and peripheral B cell development are regulated by a 9-O-acetyl sialic acid esterase. $J$ Exp Med 206, 125-138

26. Pillai, S. (2013) Rethinking mechanisms of autoimmune pathogenesis. J Autoimmun 45, 97103

27. Manzi, A. E., Sjoberg, E. R., Diaz, S. and Varki, A. (1990) Biosynthesis and turnover of Oacetyl and $\mathrm{N}$-acetyl groups in the gangliosides of human melanoma cells. J Biol Chem $\mathbf{2 6 5}$, 13091-13103

28. Hanaoka, K., Pritchett, T. J., Takasaki, S., Kochibe, N., Sabesan, S., Paulson, J. C. and Kobata, A. (1989) 4-O-acetyl-N-acetylneuraminic acid in the N-linked carbohydrate structures of equine and guinea pig alpha 2-macroglobulins, potent inhibitors of influenza virus infection. J Biol Chem 264, $9842-9849$

29. Iwersen, M., Vandamme-Feldhaus, V. and Schauer, R. (1998) Enzymatic 4-O-acetylation of $\mathrm{N}$-acetylneuraminic acid in guinea-pig liver. Glycoconj J 15, 895-904

30. Miyoshi, I., Higashi, H., Hirabayashi, Y., Kato, S. and Naiki, M. (1986) Detection of 4-Oacetyl-N-glycolylneuraminyl lactosylceramide as one of tumor-associated antigens in human colon cancer tissues by specific antibody. Mol Immunol 23, 631-638 
31. Higashihara, T., Takeshima, T., Anzai, M., Tomioka, M., Matsumoto, K., Nishida, K., Kitamura, Y., Okinaga, K. and Naiki, M. (1991) Survey of Hanganutziu and Deicher antibodies in operated patients. Int Arch Allergy Appl Immunol 95, 231-235

32. Asaoka, H., Nishinaka, S., Wakamiya, N., Matsuda, H. and Murata, M. (1992) Two chicken monoclonal antibodies specific for heterophil Hanganutziu-Deicher antigens. Immunol Lett 32, 91-96

33. Ravindranath, M. H., Higa, H. H., Cooper, E. L. and Paulson, J. C. (1985) Purification and characterization of an O-acetylsialic acid-specific lectin from a marine crab Cancer antennarius. J Biol Chem 260, 8850-8856

34. Babál, P., Pindak, F. F., Russell, L. C. and Gardner, W. A. (1999) Sialic acid-specific lectin from Tritrichomonas foetus. Biochim Biophys Acta 1428, 106-116

35. Herrler, G., Rott, R., Klenk, H. D., Müller, H. P., Shukla, A. K. and Schauer, R. (1985) The receptor-destroying enzyme of influenza $\mathrm{C}$ virus is neuraminate-O-acetylesterase. EMBO J 4, 1503-1506

36. Schultze, B., Wahn, K., Klenk, H. D. and Herrler, G. (1991) Isolated HE-protein from hemagglutinating encephalomyelitis virus and bovine coronavirus has receptor-destroying and receptor-binding activity. Virology 180, 221-228

37. Rogers, G. N., Herrler, G., Paulson, J. C. and Klenk, H. D. (1986) Influenza C virus uses 9O-acetyl-N-acetylneuraminic acid as a high affinity receptor determinant for attachment to cells. J Biol Chem 261, 5947-5951

38. Vlasak, R., Krystal, M., Nacht, M. and Palese, P. (1987) The influenza C virus glycoprotein (HE) exhibits receptor-binding (hemagglutinin) and receptor-destroying (esterase) activities. Virology 160, 419-425

39. Vlasak, R., Luytjes, W., Spaan, W. and Palese, P. (1988) Human and bovine coronaviruses recognize sialic acid-containing receptors similar to those of influenza $\mathrm{C}$ viruses. Proc Natl Acad Sci U S A 85, 4526-4529

40. Hellebø, A., Vilas, U., Falk, K. and Vlasak, R. (2004) Infectious salmon anemia virus specifically binds to and hydrolyzes 4-O-acetylated sialic acids. $J$ Virol 78, 3055-3062

41. Regl, G., Kaser, A., Iwersen, M., Schmid, H., Kohla, G., Strobl, B., Vilas, U., Schauer, R. and Vlasak, R. (1999) The hemagglutinin-esterase of mouse hepatitis virus strain $\mathrm{S}$ is a sialate-4-O-acetylesterase. J Virol 73, 4721-4727

42. Luytjes, W., Bredenbeek, P. J., Noten, A. F., Horzinek, M. C. and Spaan, W. J. (1988) Sequence of mouse hepatitis virus A59 mRNA 2: indications for RNA recombination between coronaviruses and influenza $C$ virus. Virology 166, 415-422

43. Yokomori, K., Banner, L. R. and Lai, M. M. (1991) Heterogeneity of gene expression of the hemagglutinin-esterase (HE) protein of murine coronaviruses. Virology 183, 647-657

44. Shieh, C. K., Lee, H. J., Yokomori, K., La Monica, N., Makino, S. and Lai, M. M. (1989) Identification of a new transcriptional initiation site and the corresponding functional gene $2 \mathrm{~b}$ in the murine coronavirus RNA genome. $J$ Virol 63, 3729-3736

45. Langereis, M. A., Zeng, Q., Heesters, B. A., Huizinga, E. G. and de Groot, R. J. (2012) The murine coronavirus hemagglutinin-esterase receptor-binding site: a major shift in ligand specificity through modest changes in architecture. PLoS Pathog 8, e1002492

46. Langereis, M. A., Bakkers, M. J., Deng, L., Padler-Karavani, V., Vervoort, S. J., Hulswit, R. J., van Vliet, A. L., Gerwig, G. J., de Poot, S. A., Boot, W., van Ederen, A. M., Heesters, B. A., van der Loos, C. M., van Kuppeveld, F. J., Yu, H., Huizinga, E. G., Chen, X., Varki, A., 
Kamerling, J. P. and de Groot, R. J. (2015) Complexity and Diversity of the Mammalian Sialome Revealed by Nidovirus Virolectins. Cell Rep 11, 1966-1978

47. Wasik, B. R., Barnard, K. N., Ossiboff, R. J., Khedri, Z., Feng, K. H., Yu, H., Chen, X., Perez, D. R., Varki, A. and Parrish, C. R. (2017) Distribution of O-Acetylated Sialic Acids among Target Host Tissues for Influenza Virus. mSphere 2,

48. Tangvoranuntakul, P., Gagneux, P., Diaz, S., Bardor, M., Varki, N., Varki, A. and Muchmore, E. (2003) Human uptake and incorporation of an immunogenic nonhuman dietary sialic acid. Proc Natl Acad Sci U S A 100, 12045-12050

49. Miyake, M., Ito, M., Hitomi, S., Ikeda, S., Taki, T., Kurata, M., Hino, A., Miyake, N. and Kannagi, R. (1988) Generation of two murine monoclonal antibodies that can discriminate $\mathrm{N}$-glycolyl and N-acetyl neuraminic acid residues of GM2 gangliosides. Cancer Res $\mathbf{4 8}$, 6154-6160

50. Tai, T., Kawashima, I., Furukawa, K. and Lloyd, K. O. (1988) Monoclonal antibody R24 distinguishes between different N-acetyl- and N-glycolylneuraminic acid derivatives of ganglioside GD3. Arch Biochem Biophys 260, 51-55

51. Ohashi, Y., Sasabe, T., Nishida, T., Nishi, Y. and Higashi, H. (1983) Hanganutziu-Deicher heterophile antigen in human retinoblastoma cells. Am J Ophthalmol 96, 321-325

52. Higashi, H., Sasabe, T., Fukui, Y., Maru, M. and Kato, S. (1988) Detection of gangliosides as N-glycolylneuraminic acid-specific tumor-associated Hanganutziu-Deicher antigen in human retinoblastoma cells. Jpn J Cancer Res 79, 952-956

53. Higashi, H., Nishi, Y., Fukui, Y., Ikuta, K., Ueda, S., Kato, S., Fujita, M., Nakano, Y., Taguchi, T. and Sakai, S. (1984) Tumor-associated expression of glycosphingolipid Hanganutziu-Deicher antigen in human cancers. Gan 75, 1025-1029

54. Higashi, H., Hirabayashi, Y., Fukui, Y., Naiki, M., Matsumoto, M., Ueda, S. and Kato, S. (1985) Characterization of N-glycolylneuraminic acid-containing gangliosides as tumorassociated Hanganutziu-Deicher antigen in human colon cancer. Cancer Res 45, 3796-3802

55. Hirabayashi, Y., Kasakura, H., Matsumoto, M., Higashi, H., Kato, S., Kasai, N. and Naiki, M. (1987) Specific expression of unusual GM2 ganglioside with Hanganutziu-Deicher antigen activity on human colon cancers. Jpn J Cancer Res 78, 251-260

56. Diaz, S. L., Padler-Karavani, V., Ghaderi, D., Hurtado-Ziola, N., Yu, H., Chen, X., Brinkman-Van der Linden, E. C., Varki, A. and Varki, N. M. (2009) Sensitive and specific detection of the non-human sialic Acid N-glycolylneuraminic acid in human tissues and biotherapeutic products. PLoS One 4, e4241

57. Day, C. J., Paton, A. W., Higgins, M. A., Shewell, L. K., Jen, F. E., Schulz, B. L., Herdman, B. P., Paton, J. C. and Jennings, M. P. (2017) Structure aided design of a Neu5Gc specific lectin. Sci Rep 7, 1495

58. Shewell, L. K., Wang, J. J., Paton, J. C., Paton, A. W., Day, C. J. and Jennings, M. P. (2018) Detection of N-glycolylneuraminic acid biomarkers in sera from patients with ovarian cancer using an engineered N-glycolylneuraminic acid-specific lectin SubB2M. Biochem Biophys Res Commun

59. Wang, J., Shewell, L. K., Paton, A. W., Paton, J. C., Day, C. J. and Jennings, M. P. (2018) Specificity and utility of SubB2M, a new N-glycolylneuraminic acid lectin. Biochem Biophys Res Commun 500, 765-771

60. Samraj, A. N., Pearce, O. M., Läubli, H., Crittenden, A. N., Bergfeld, A. K., Banda, K., Gregg, C. J., Bingman, A. E., Secrest, P., Diaz, S. L., Varki, N. M. and Varki, A. (2015) A 
red meat-derived glycan promotes inflammation and cancer progression. Proc Natl Acad Sci $U S$ A 112, 542-547

61. Nicholls, J. M., Bourne, A. J., Chen, H., Guan, Y. and Peiris, J. S. (2007) Sialic acid receptor detection in the human respiratory tract: evidence for widespread distribution of potential binding sites for human and avian influenza viruses. Respir Res 8, 73

62. Jia, N., Byrd-Leotis, L., Matsumoto, Y., Gao, C., Wein, A. N., Lobby, J. L., Kohlmeier, J. E., Steinhauer, D. A. and Cummings, R. D. (2020) The Human Lung Glycome Reveals Novel Glycan Ligands for Influenza A Virus. Sci Rep 10, 5320

63. Lehmann, F., Tiralongo, E. and Tiralongo, J. (2006) Sialic acid-specific lectins: occurrence, specificity and function. Cell Mol Life Sci 63, 1331-1354

64. Tateno, H., Winter, H. C. and Goldstein, I. J. (2004) Cloning, expression in Escherichia coli and characterization of the recombinant Neu5Acalpha2,6Galbeta1,4GlcNAc-specific highaffinity lectin and its mutants from the mushroom Polyporus squamosus. Biochem $J \mathbf{3 8 2}$, 667-675

65. Kadirvelraj, R., Grant, O. C., Goldstein, I. J., Winter, H. C., Tateno, H., Fadda, E. and Woods, R. J. (2011) Structure and binding analysis of Polyporus squamosus lectin in complex with the Neu5Ac \{alpha\}2-6Gal \{beta\}1-4GlcNAc human-type influenza receptor. Glycobiology 21, 973-984

66. Powell, L. D. and Varki, A. (1994) The oligosaccharide binding specificities of CD22beta, a sialic acid-specific lectin of B cells. J Biol Chem 269, 10628-10636

67. Sjoberg, E. R., Powell, L. D., Klein, A. and Varki, A. (1994) Natural ligands of the B cell adhesion molecule CD22 beta can be masked by 9-O-acetylation of sialic acids. $J$ Cell Biol 126, 549-562

68. Powell, L. D., Sgroi, D., Sjoberg, E. R., Stamenkovic, I. and Varki, A. (1993) Natural ligands of the B cell adhesion molecule CD22beta carry N-linked oligosaccharides with alpha-2,6linked sialic acids that are required for recognition. $J$ Biol Chem 268, 7019-7027

69. Kelm, S., Pelz, A., Schauer, R., Filbin, M. T., Tang, S., de Bellard, M. E., Schnaar, R. L., Mahoney, J. A., Hartnell, A. and Bradfield, P. (1994) Sialoadhesin, myelin-associated glycoprotein and CD22 define a new family of sialic acid-dependent adhesion molecules of the immunoglobulin superfamily. Curr Biol 4, 965-972

70. Brinkman-Van der Linden, E. C., Sjoberg, E. R., Juneja, L. R., Crocker, P. R., Varki, N. and Varki, A. (2000) Loss of N-glycolylneuraminic acid in human evolution. Implications for sialic acid recognition by siglecs. J Biol Chem 275, 8633-8640

71. Atack, J. M., Day, C. J., Poole, J., Brockman, K. L., Bakaletz, L. O., Barenkamp, S. J. and Jennings, M. P. (2018) The HMW2 adhesin of non-typeable Haemophilus influenzae is a human-adapted lectin that mediates high-affinity binding to 2-6 linked N-acetylneuraminic acid glycans. Biochem Biophys Res Commun 503, 1103-1107

72. Shibuya, N., Goldstein, I. J., Broekaert, W. F., Nsimba-Lubaki, M., Peeters, B. and Peumans, W. J. (1987) The elderberry (Sambucus nigra L.) bark lectin recognizes the Neu5Ac(alpha 2-6)Gal/GalNAc sequence. J Biol Chem 262, 1596-1601

73. Yamashita, K., Umetsu, K., Suzuki, T. and Ohkura, T. (1992) Purification and characterization of a Neu5Ac alpha 2-->6Gal beta 1-->4GlcNAc and HSO3(-)-->6Gal beta 1-->GlcNAc specific lectin in tuberous roots of Trichosanthes japonica. Biochemistry 31, 11647-11650

74. Lewis, A. L., Nizet, V. and Varki, A. (2004) Discovery and characterization of sialic acid Oacetylation in group B Streptococcus. Proc Natl Acad Sci U S A 101, 11123-11128 
75. Gurung, M. K., Raeder, I. L., Altermark, B. and Smalas, A. O. (2013) Characterization of the sialic acid synthase from Aliivibrio salmonicida suggests a novel pathway for bacterial synthesis of 7-O-acetylated sialic acids. Glycobiology 23, 806-819

76. Wipfler, D., Srinivasan, G. V., Sadick, H., Kniep, B., Arming, S., Willhauck-Fleckenstein, M., Vlasak, R., Schauer, R. and Schwartz-Albiez, R. (2011) Differentially regulated expression of 9-O-acetyl GD3 (CD60b) and 7-O-acetyl-GD3 (CD60c) during differentiation and maturation of human T and B lymphocytes. Glycobiology 21, 1161-1172

77. Vandamme-Feldhaus, V. and Schauer, R. (1998) Characterization of the enzymatic 7-Oacetylation of sialic acids and evidence for enzymatic O-acetyl migration from C-7 to C-9 in bovine submandibular gland. J Biochem 124, 111-121

78. Schauer, R. (1987) Sialic acids: metabolism of O-acetyl groups. Methods Enzymol 138, 611626

79. Stewart, A. D., Batty, J. and Harkiss, G. D. (1978) Genetic variation in plasma thyroxine levels and minimal metabolic rates of the mouse, Mus musculus. Genet Res 31, 303-306

80. Langereis, M. A., van Vliet, A. L., Boot, W. and de Groot, R. J. (2010) Attachment of mouse hepatitis virus to $\mathrm{O}$-acetylated sialic acid is mediated by hemagglutinin-esterase and not by the spike protein. J Virol 84, 8970-8974

81. Klausegger, A., Strobl, B., Regl, G., Kaser, A., Luytjes, W. and Vlasak, R. (1999) Identification of a coronavirus hemagglutinin-esterase with a substrate specificity different from those of influenza $\mathrm{C}$ virus and bovine coronavirus. J Virol 73, 3737-3743

82. Langereis, M. A., Zeng, Q., Gerwig, G. J., Frey, B., von Itzstein, M., Kamerling, J. P., de Groot, R. J. and Huizinga, E. G. (2009) Structural basis for ligand and substrate recognition by torovirus hemagglutinin esterases. Proc Natl Acad Sci U S A 106, 15897-15902

83. Schultze, B., Gross, H. J., Brossmer, R. and Herrler, G. (1991) The S protein of bovine coronavirus is a hemagglutinin recognizing 9-O-acetylated sialic acid as a receptor determinant. J Virol 65, 6232-6237

84. Schultze, B. and Herrler, G. (1992) Bovine coronavirus uses N-acetyl-9-O-acetylneuraminic acid as a receptor determinant to initiate the infection of cultured cells. J Gen Virol 73, 901906

85. Kemminer, S. E., Conradt, H. S., Nimtz, M., Sagi, D., Peter-Katalinić, J., Diekmann, O., Drmić, I. and Müthing, J. (2001) Production and molecular characterization of clinical phase i anti-melanoma mouse IgG3 monoclonal antibody R24. Biotechnol Prog 17, 809-821

86. Heiner, J. P., Miraldi, F., Kallick, S., Makley, J., Neely, J., Smith-Mensah, W. H. and Cheung, N. K. (1987) Localization of GD2-specific monoclonal antibody 3F8 in human osteosarcoma. Cancer Res 47, 5377-5381

87. Manzke, O., Russello, O., Leenen, C., Diehl, V., Bohlen, H. and Berthold, F. (2001) Immunotherapeutic strategies in neuroblastoma: antitumoral activity of deglycosylated Ricin A conjugated anti-GD2 antibodies and anti-CD3xanti-GD2 bispecific antibodies. Med Pediatr Oncol 36, 185-189

88. Castel, V., Segura, V. and Cañete, A. (2010) Treatment of high-risk neuroblastoma with antiGD2 antibodies. Clin Transl Oncol 12, 788-793

89. Kawashima, I., Tada, N., Ikegami, S., Nakamura, S., Ueda, R. and Tai, T. (1988) Mouse monoclonal antibodies detecting disialogangliosides on mouse and human $\mathrm{T}$ lymphomas. Int J Cancer 41, 267-274 
90. Sjoberg, E. R., Manzi, A. E., Khoo, K. H., Dell, A. and Varki, A. (1992) Structural and immunological characterization of O-acetylated GD2. Evidence that GD2 is an acceptor for ganglioside O-acetyltransferase in human melanoma cells. J Biol Chem 267, 16200-16211

91. Vinson, M., Strijbos, P. J., Rowles, A., Facci, L., Moore, S. E., Simmons, D. L. and Walsh, F. S. (2001) Myelin-associated glycoprotein interacts with ganglioside GT1b. A mechanism for neurite outgrowth inhibition. J Biol Chem 276, 20280-20285

92. Ito, H., Ishida, H., Waki, H., Ando, S. and Kiso, M. (1999) Total synthesis of a cholinergic neuron-specific ganglioside GT1a alpha: a high affinity ligand for myelin-associated glycoprotein (MAG). Glycoconj J 16, 585-598

93. Hashimoto, N., Ito, S., Tsuchida, A., Bhuiyan, R. H., Okajima, T., Yamamoto, A., Furukawa, K., Ohmi, Y. and Furukawa, K. (2019) The ceramide moiety of disialoganglioside (GD3) is essential for GD3 recognition by the sialic acid-binding lectin SIGLEC7 on the cell surface. J Biol Chem

94. Ravindranaths, M. H., Paulson, J. C. and Irie, R. F. (1988) Human melanoma antigen Oacetylated ganglioside GD3 is recognized by Cancer antennarius lectin. J Biol Chem 263, 2079-2086

95. Yagi, F., Miyamoto, M., Abe, T., Minami, Y., Tadera, K. and Goldstein, I. J. (1997) Purification and carbohydrate-binding specificity of Agrocybe cylindracea lectin. Glycoconj $J$ 14, 281-288

96. Monsigny, M., Roche, A. C., Sene, C., Maget-Dana, R. and Delmotte, F. (1980) Sugar-lectin interactions: how does wheat-germ agglutinin bind sialoglycoconjugates. Eur J Biochem 104, 147-153

97. Kim, D. S., Son, K. Y., Koo, K. M., Kim, J. Y., Alfajaro, M. M., Park, J. G., Hosmillo, M., Soliman, M., Baek, Y. B., Cho, E. H., Lee, J. H., Kang, M. I., Goodfellow, I. and Cho, K. O. (2016) Porcine Sapelovirus Uses $\alpha 2,3$-Linked Sialic Acid on GD1a Ganglioside as a Receptor. J Virol 90, 4067-4077

98. Benktander, J., Barone, A., Johansson, M. M. and Teneberg, S. (2018) Helicobacter pylori SabA binding gangliosides of human stomach. Virulence 9, 738-751

99. Iwamori, M., Takamizawa, K., Momoeda, M., Iwamori, Y. and Taketani, Y. (2008) Gangliosides in human, cow and goat milk, and their abilities as to neutralization of cholera toxin and botulinum type A neurotoxin. Glycoconj $J$ 25, 675-683

100. Halpern, J. L. and Loftus, A. (1993) Characterization of the receptor-binding domain of tetanus toxin. J Biol Chem 268, 11188-11192

101. Louch, H. A., Buczko, E. S., Woody, M. A., Venable, R. M. and Vann, W. F. (2002) Identification of a binding site for ganglioside on the receptor binding domain of tetanus toxin. Biochemistry 41, 13644-13652

102. Chen, C., Fu, Z., Kim, J. J., Barbieri, J. T. and Baldwin, M. R. (2009) Gangliosides as high affinity receptors for tetanus neurotoxin. J Biol Chem 284, 26569-26577

103. Chen, C., Baldwin, M. R. and Barbieri, J. T. (2008) Molecular basis for tetanus toxin coreceptor interactions. Biochemistry 47, 7179-7186

104. Varki, A. (1992) Diversity in the sialic acids. Glycobiology 2, 25-40

105. Mandal, C. and Basu, S. (1987) An unique specificity of a sialic acid binding lectin AchatininH, from the hemolymph of Achatina fulica snail. Biochem Biophys Res Commun 148, 795-801

106. Muchmore, E. A. and Varki, A. (1987) Selective inactivation of influenza C esterase: a probe for detecting 9-O-acetylated sialic acids. Science 236, 1293-1295 
107. Zimmer, G., Reuter, G. and Schauer, R. (1992) Use of influenza C virus for detection of 9O-acetylated sialic acids on immobilized glycoconjugates by esterase activity. Eur J Biochem 204, 209-215

108. Klein, A., Krishna, M., Varki, N. M. and Varki, A. (1994) 9-O-acetylated sialic acids have widespread but selective expression: analysis using a chimeric dual-function probe derived from influenza C hemagglutinin-esterase. Proc Natl Acad Sci U S A 91, 7782-7786

109. Martin, L. T., Verhagen, A. and Varki, A. (2003) Recombinant influenza C hemagglutininesterase as a probe for sialic acid 9-O-acetylation. Methods Enzymol 363, 489-498

110. Varki, A. and Diaz, S. (1984) The release and purification of sialic acids from glycoconjugates: methods to minimize the loss and migration of O-acetyl groups. Anal Biochem 137, 236-247

111. Higa, H. H., Manzi, A. and Varki, A. (1989) O-acetylation and de-O-acetylation of sialic acids. Purification, characterization, and properties of a glycosylated rat liver esterase specific for 9-O-acetylated sialic acids. J Biol Chem 264, 19435-19442

112. Hara, S., Yamaguchi, M., Takemori, Y., Furuhata, K., Ogura, H. and Nakamura, M. (1989) Determination of mono-O-acetylated $\mathrm{N}$-acetylneuraminic acids in human and rat sera by fluorometric high-performance liquid chromatography. Anal Biochem 179, 162-166

113. Padler-Karavani, V., Tremoulet, A. H., Yu, H., Chen, X., Burns, J. C. and Varki, A. (2013) A simple method for assessment of human anti-Neu5Gc antibodies applied to Kawasaki disease. PLoS One 8, e 58443 
bioRxiv preprint doi: https://doi.org/10.1101/2021.05.28.446202; this version posted May 28, 2021. The copyright holder for this preprint (which

was not certified by peer review) is the author/funder, who has granted bioRxiv a license to display the preprint in perpetuity. It is made available under aCC-BY-NC-ND 4.0 International license.

\begin{abstract}
Abbreviations
SGRP (Sialoglycan Recognition Probe); Sia (Sialic Acids); Siglec (Sialic acid-binding immunoglobulin-type lectins); YenB (Yersinia enterocolitica, toxin subunit B); HE (Hemagglutinin Esterase); HA (Hemagglutinins Adhesins); Neu5Ac (N-Acetylneuraminic acid); Neu5Gc (N-Glycolylneuraminic acid);
\end{abstract}




\section{Tables}

Table 1. Sialoglycan Recognition Probes and their specificities. Table representing the classes of Sialoglycan Recognition Probes (SGRPs), their presumed binding specificities, the most appropriate molecules as observed by assessment of sialoglycan binding by various glycomic methods, their experimentally confirmed sialoglycan preferences, and defining SGRP nomenclature suggesting class of probe along with source of probe as mentioned in superscripts. The names of probes ending with NB represent the non-binding variant of SGRPs.

\begin{tabular}{|c|c|c|c|c|c|}
\hline \multirow{2}{*}{$\begin{array}{c}\text { Class of SialoGlycan } \\
\text { Recognition Probe }\end{array}$} & \multirow{2}{*}{ Sialoglycans classes } & \multirow{2}{*}{$\begin{array}{c}\text { Identified } \\
\text { molecule }\end{array}$} & \multirow{2}{*}{$\begin{array}{l}\text { Sialoglycan } \\
\text { preferences }\end{array}$} & \multicolumn{2}{|c|}{ SGRPs Nomenclature } \\
\hline & & & & Binding & Non-binding \\
\hline SGRP1 & All Sialic Acids (Sias) & YenB & All Sias & SGRP1 YenB & $\mathrm{SGRP} 1^{Y e n B} \mathrm{NB}$ \\
\hline SGRP2 & 5-N-acetylneuraminic acid (Neu5Ac) & PltB & All Neu5Ac glycans & $\mathrm{SGRP2} 2^{P l t B}$ & $\mathrm{SGRP} 2^{P t t B} \mathrm{NB}$ \\
\hline SGRP3 & $\alpha-2-3$ Linked Sialic Acids ( $\alpha-2-3$ Sias $)$ & Hsa $_{\mathrm{BR}}$ & All $\alpha 2-3$-linked Sias & SGRP3 ${ }^{H s a}$ & SGRP3 ${ }^{H s a} \mathrm{NB}$ \\
\hline SGRP4 & 4-O-Acetylated Sialic Acids (4-OAc Sias) & MHV & 4-OAcetylated Neu5Ac & SGRP4 ${ }^{M H V}$ & $\mathrm{SGRP} 4^{M H V} \mathrm{NB}$ \\
\hline SGRP5 & 5-N-glycolylneuraminic acid (Neu5Gc) & $\operatorname{IgY}$ & All Neu5Gc glycans & SGRP5 $\operatorname{Ig} Y$ & $\mathrm{SGRP}^{\operatorname{Ig} Y} \mathrm{NB}$ \\
\hline SGRP6 & $\alpha-2-6$ Linked Sialic Acids ( $\alpha-2-6$ Sias) & SNA & All $\alpha 2-6$ Linked Sia & $\mathrm{SGRP} 6^{S N A}$ & N/A \\
\hline SGRP7 & 7-O-Acetylated Sialic Acids (7-OAc Sias) & $\mathrm{BCoV}$ & 7-9-di O-Acetylated Sia & SGRP7 ${ }^{B C o V}$ & $\mathrm{SGRP} 7^{B C o V} \mathrm{NB}$ \\
\hline SGRP8 & $\alpha-2-8$ Linked Di-Sialic Acid linkage & $\mathrm{TeT}$ & Disialic linkages & SGRP8 ${ }^{T e T}$ & SGRP8 ${ }^{T e T} \mathrm{NB}$ \\
\hline SGRP9 & 9-O-Acetylated Sialic Acids (9-OAc Sias) & PToV & All 9-O-Acetylated Sia & SGRP9PToV & SGRP9 ${ }^{P T o}$ NB \\
\hline
\end{tabular}




\section{Figure Legends}

\section{Figure 1. Sialoglycan Microarray Binding Studies of Proposed Sialoglycan Recognizing} Probes (SGRPs). Heatmap analysis of SGRPs (see Table 1 for details of nomenclature) binding to mammalian (sialo) glycans; SGRP1 ${ }^{\text {YenB }}(30 \mu \mathrm{g} / \mathrm{ml})$, SGRP2 ${ }^{\text {PltB }}$ (30 $\left.\mu \mathrm{g} / \mathrm{ml}\right)$, SGRP3 ${ }^{\text {Hsa }}$ (30 $\mu \mathrm{g} / \mathrm{ml}), \operatorname{SGRP}^{M H V}(30 \mu \mathrm{g} / \mathrm{ml}), \operatorname{SGRP}^{\operatorname{Ig} Y}(20 \mu \mathrm{g} / \mathrm{ml}), \operatorname{SGRP6}^{S N A}(20 \mu \mathrm{g} / \mathrm{ml}), \mathrm{SGRP}^{B C o V}(60$ $\mu \mathrm{g} / \mathrm{ml}), \operatorname{SGRP}^{T e T}(30 \mu \mathrm{g} / \mathrm{ml})$ and SGRP9 ${ }^{P T o V}(30 \mu \mathrm{g} / \mathrm{ml})$ in microarray experiments. SGRPs binding efficiencies are displayed in red for highest binding (saturated or 100\%), blue for minimum or no binding $(0 \%)$ and intermediate binding represented by colors ranging between blue and red. Ranks; Red (100\%, or maximum), Blue ( $0 \%$, or minimum). Markedly reduced or absent binding was seen with the no-binding control probes studied simultaneously (See Supplementary Figure S4).

Figure 2. Specific binding of SGRPs to sialoglycans in mammalian sera. The figure presents SGRPs binding of sialoglycans in biological samples, here represented by mammalian sera from mouse, rat, rabbit, guinea pig, goat, sheep, horse, human and $\mathrm{Cmah}^{-/-}$mouse. Panels A-I (except panel $\mathrm{H}$ ) show binding efficiency of SGRPs and their nonbinding controls towards sera in concentration-dependent manner, as marked in individual panels. Panel $\mathrm{H}$ shows binding of SGRP8TeT and SGRP8TeTNB of purified gangliosides at concentrations mentioned in panel. Panel J demonstrates the 7,9-diOAc-Sia-specific binding of SGRP7BCoV with bovine submaxillary mucin while its mutant SGRP7BCoVNB shows no affinity towards BSM. Binding of biotinylated SGRPs was detected and developed using Avidin-HRP (1:1500). ELISA for each SGRP pair of was performed in same experiment/conditions and validity of binding was confirmed by background signals wells treated only with secondary antibody (Avidin-HRP), not shown here.

Figure 3. Specific recognition of sialoglycoproteins in mammalian sera by SGRPs in western blots. Mammalian sera from mouse (1), rat (2), rabbit (3), guinea pig (4), goat (5), sheep (6), horse (7), human (8) and $\mathrm{Cmah}^{-/-}$mouse (9), were subjected to SDS-PAGE under conditions that protect labile O-acetyl groups immunoblotted and probed with SGRPs. M in each blot represents the molecular weight marker. Panels A \& B show blots interrogated with SGRP1YenB and SGRP1YenBNB (both at $1 \mu \mathrm{g} / \mathrm{ml}$ ); panels C \& D show blots interrogated with $1 \mu \mathrm{g} / \mathrm{ml} \mathrm{SGRP2PltB}$ and SGRP2PltBNB; panels E \& F represent SGRP3Hsa and SGRP3HsaNB (both at $1 \mu \mathrm{g} / \mathrm{ml}$ ); 
panels G \& H shows blots probed with $1 \mu \mathrm{g} / \mathrm{ml}$ of SGRP4MHV and SGRP4MHVNB; panels I \& J shows blot probed with $0.33 \mu \mathrm{g} / \mathrm{ml}$ SGRP5IgY and SGRP5IgYNB; panels $\mathrm{K}$ is blot interrogated by $1 \mu \mathrm{g} / \mathrm{ml}$ SGRP6SNA; panels L \& $\mathrm{M}$ show blots probed by $1 \mu \mathrm{g} / \mathrm{ml}$ SGRP7BCoV and SGRP7BCoVNB, and panels $\mathrm{N} \& \mathrm{O}$ represent blots interrogated with $2 \mu \mathrm{g} / \mathrm{ml}$ SGRP9PToV and SGRP9PToVNB. Binding of biotinylated SGRPs was detectable and developed using Streptavidin IRDye 680 (1:10,000). Analysis was done on a LiCor Odyssey infrared imager. Both blots for each SGRP set were detected at the same time using the same conditions.

Figure 4. Histological analysis of frozen mouse tissues by SGRPs: The panel shows examples of binding patterns observed with each SGRP; binding indicated by red fluorescent staining. In situ sialoglycan detection ability of SGRP1 $1^{\text {YenB }}(5 \mu \mathrm{g} / \mathrm{ml})$ is demonstrated using frozen kidney sections from WT and $\mathrm{Cmah}^{-/-}$mouse (Panels $A \& B$ ) and showing binding on glomeruli and blood vessels while the nonbinding variant $\mathrm{SGRP} 1^{Y e n B} \mathrm{NB}$ shows no binding to tissue sections as expected (Panel C). Panels D, E\& G, H show SGRP2 ${ }^{\text {PltB }}(5 \mu \mathrm{g} / \mathrm{ml})$ and SGRP3 ${ }^{H s a}(3 \mu \mathrm{g} / \mathrm{ml})$ recognition of glomeruli and vessels in kidney sections from WT and $\mathrm{Cmah}^{-/-}$mouse, while Panel $F \& I$ show no binding of SGRP2 ${ }^{P l t B} \mathrm{NB}$ and $\mathrm{SGRP} 3^{H s a} \mathrm{NB}$ to tissue sections as expected. SGRP4 ${ }^{M H V}(5 \mu \mathrm{g} / \mathrm{ml})$ shows selective detection of its ligand in mucins in the pancreatic acini more prominently in $\mathrm{Cmah}^{-/-}$mouse (Panel K) than in WT (Panel J), while SGRP4 ${ }^{M H V} \mathrm{NB}$ shows no evidence of binding at all as expected (Panel L). Panel $M(1 \mu \mathrm{g} / \mathrm{ml})$ shows SGRP5 ${ }^{\operatorname{Ig} Y}$ binding to WT mouse pancreas, while Panel $N$ shows no binding, with the expected lack of SGRP5 ${ }^{\operatorname{Ig} Y}$ ligands in $\mathrm{Cmah}^{-/}$mouse. SGRP5 ${ }^{\operatorname{Ig} Y} \mathrm{NB}$ does not bind to tissue sections (Panel O) as expected. Panels $P$ $\& Q$ represent SGRP6 ${ }^{S N A}(2 \mu \mathrm{g} / \mathrm{ml})$ binding to blood vessels in WT and $\mathrm{Cmah}^{-/-}$mouse pancreas. Panel $R$ represents a control experiment; tissue stained with secondary antibody Cy3-SA (1:500) only. Panel $S \& T$ shows that SGRP7 ${ }^{B C o V}(10 \mu \mathrm{g} / \mathrm{ml})$ binds to blood vessels in brains of WT and $\mathrm{Cmah}^{-/-}$mouse and Panel $U$ demonstrate no binding of SGRP7 ${ }^{\mathrm{BCoV}} \mathrm{NB}$ in same tissue as expected. Panel $V \& W$ suggest that SGRP8 ${ }^{T e T}(5 \mu \mathrm{g} / \mathrm{ml})$ binds to its ligand predominantly present in WT and $\mathrm{Cmah}^{-/}$mouse brains, while SGRP8 ${ }^{T e T} \mathrm{NB}$ doesn't show any binding on tissue section (Panel $X)$. Panels $Y \& Z$ represent SGRP9 ${ }^{P T o V}(5 \mu \mathrm{g} / \mathrm{ml})$ binding to livers of WT and more prominently in livers of $\mathrm{Cmah}^{-/}$mouse. Panel Z1 shows SGRP9 ${ }^{P T o V}$ NB doesn't binding to tissue, as expected. 
Figure 5. SGRPs recognize Specific sialoglycans on mammalian erythrocytes: The panels, as marked in figure represent SGRPs binding with RBCs for 9 animal sources. The mean fluorescence intensities (MFI) values for SGRPs; binding and non-binding versions are mentioned for each type of erythrocyte. Panel A represents SGRP1 ${ }^{\text {YenB }}(5 \mu \mathrm{g} / \mathrm{ml})$ binding with all tested RBCs. Panel B represents SGRP2 ${ }^{P l t B}(5 \mu \mathrm{g} / \mathrm{ml})$ binding with RBCs in accordance to the fractional representation of Neu5Ac-sialoglycans. Panel C shows SGRP3 ${ }^{H s a}(5 \mu \mathrm{g} / \mathrm{ml})$ binding with all RBCs, while Panel D suggest SGRP4 ${ }^{M H V}$ does not specifically bind to RBCs, suggesting lack of its ligand. SGRP5 ${ }^{\operatorname{Ig} Y}$ $(2 \mu \mathrm{g} / \mathrm{ml})$ binds prominently with all RBCs except rat and guinea pig (Panel E) while SGRP6 ${ }^{\text {SNA }}$ $(2 \mu \mathrm{g} / \mathrm{ml})$ lacks binding to goat, sheep and horse RBCs (Panel F). Panel G suggest the RBCs do not express SGRP7 ${ }^{B C o V}$ 's specific ligand 7,9-diOAc-Sias, resulting into negligible binding of the probe $(5 \mu \mathrm{g} / \mathrm{ml})$. Panel $\mathrm{H}$ shows SGRP9 ${ }^{P T o V}(5 \mu \mathrm{g} / \mathrm{ml})$ affinity towards surface glycans terminating in 9-OAc-Sias on RBC surfaces. Panel I represent significantly enhanced binding of SGRP8TeT $(5 \mu \mathrm{g} / \mathrm{ml})$ towards disialogangliosides expressed by GD3 transfected $\mathrm{CHO}$ cell in comparison to native CHOK1 cells. Panel J is the comprehensive heat map representation of SGRP's binding to mammalian erythrocytes; color red and blue represent maximum and minimum binding efficiencies respectively. 
bioRxiv preprint doi: https://doi.org/10.1101/2021.05.28.446202; this version posted May 28, 2021. The copyright holder for this preprint (which was not certified by peer review) is the author/funder, who has granted bioRxiv a license to display the preprint in perpetuity. It is made available under aCC-BY-NC-ND 4.0 International license.

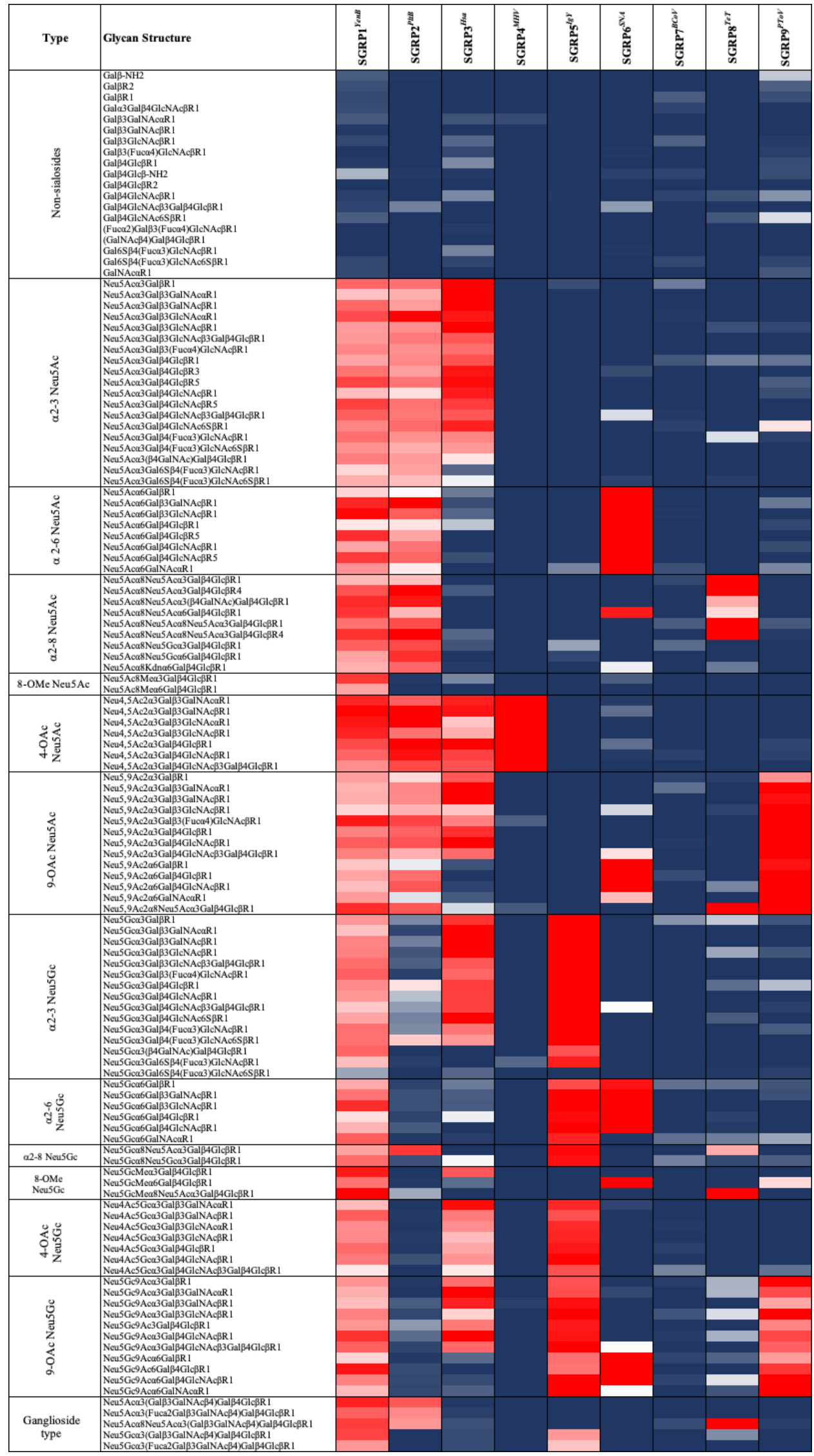

Figure 1 
bioRxiv preprint doi: https://doi.org/10.1101/2021.05.28.446202; this version posted May 28, 2021. The copyright holder for this preprint (which was not certified by peer review) is the author/funder, who has granted bioRxiv a license to display the preprint in perpetuity. It is made available under aCC-BY-NC-ND 4.0 International license.

A

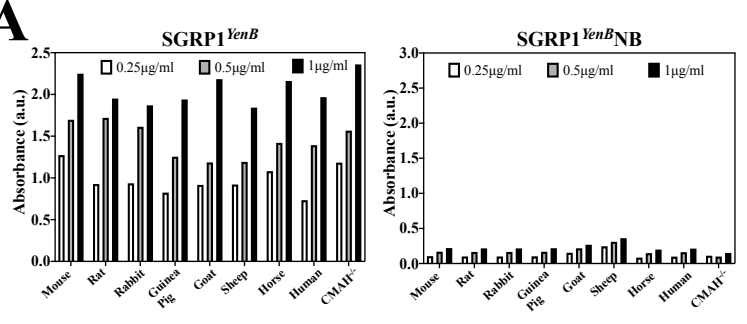

C

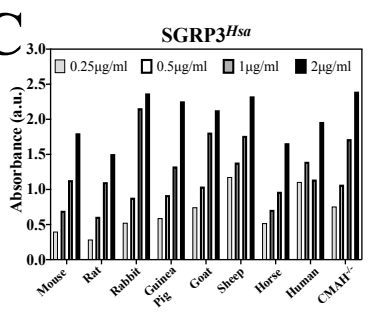

E
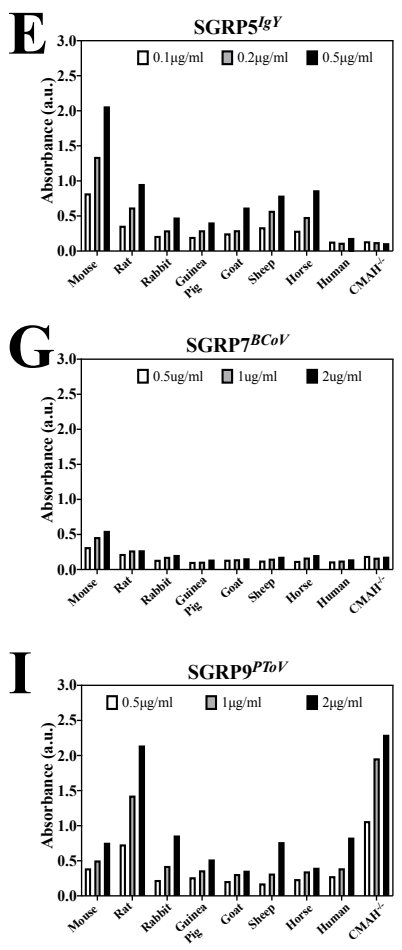

B
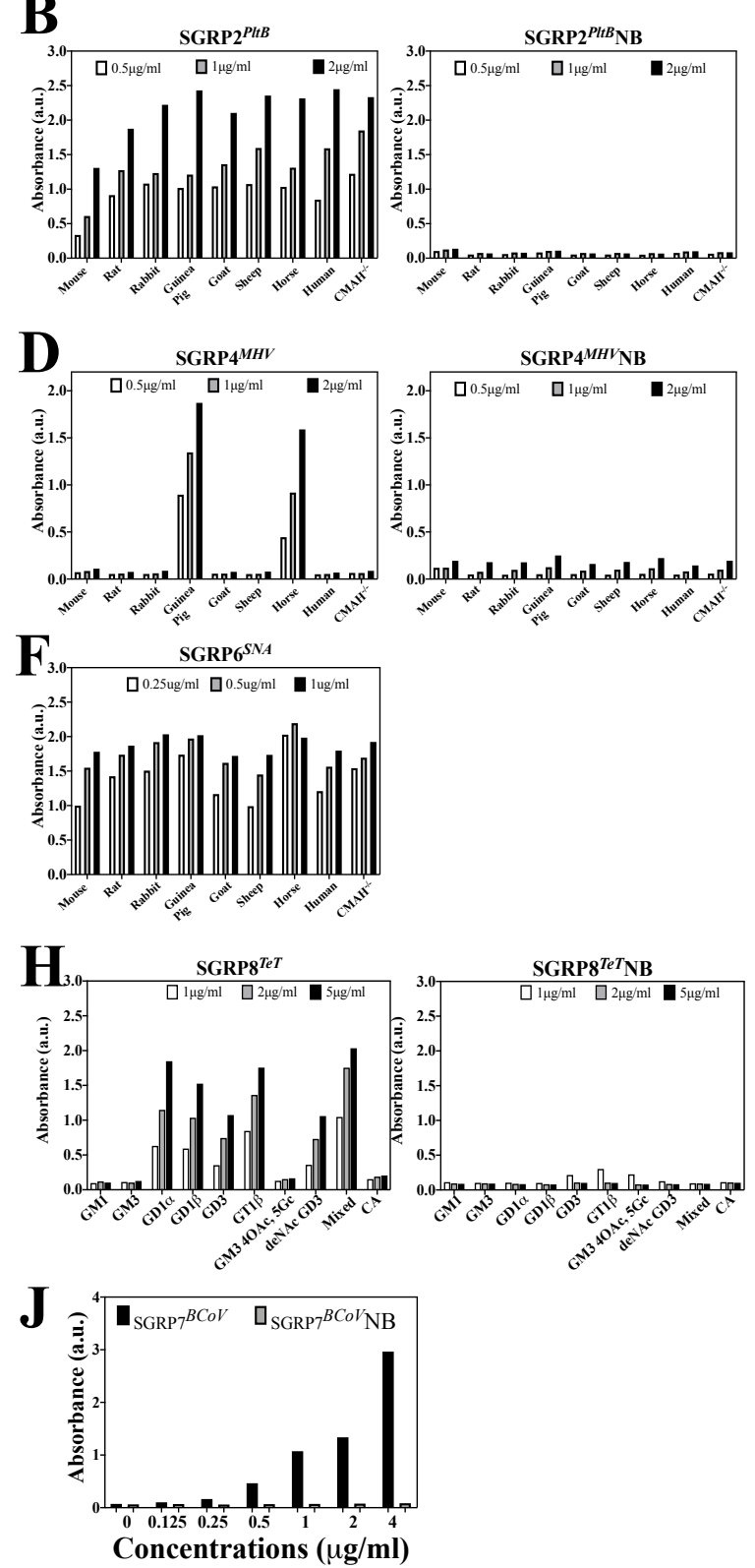

Figure 2 

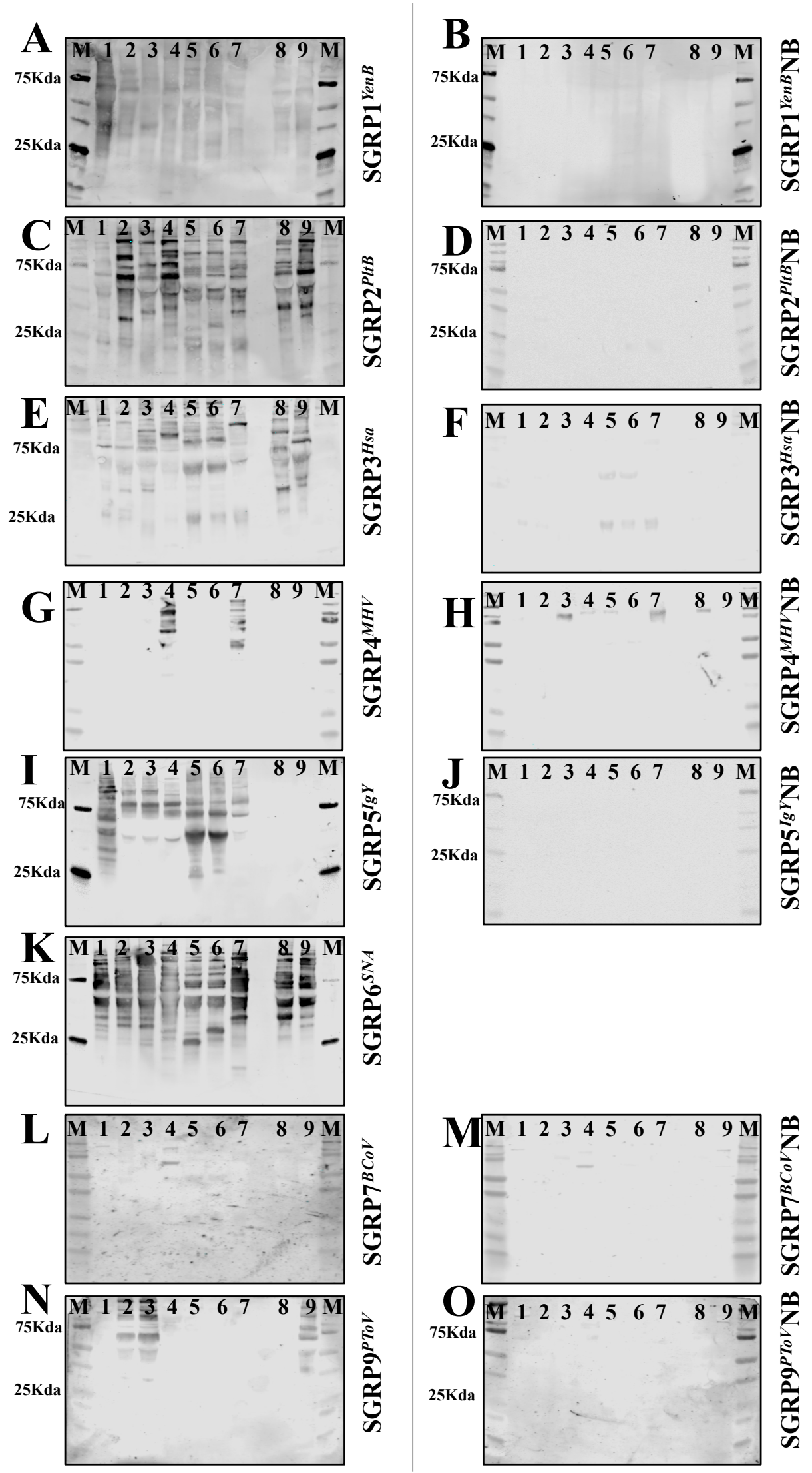

\section{Figure 3}


bioRxiv preprint doi: https://doi.org/10.1101/2021.05.28.446202; this version posted May 28, 2021. The copyright holder for this preprint (which

was not certified by peer review) is the author/funder, who has granted bioRxiv a license to display the preprint in perpetuity. It is made available under aCC-BY-NC-ND 4.0 International license.

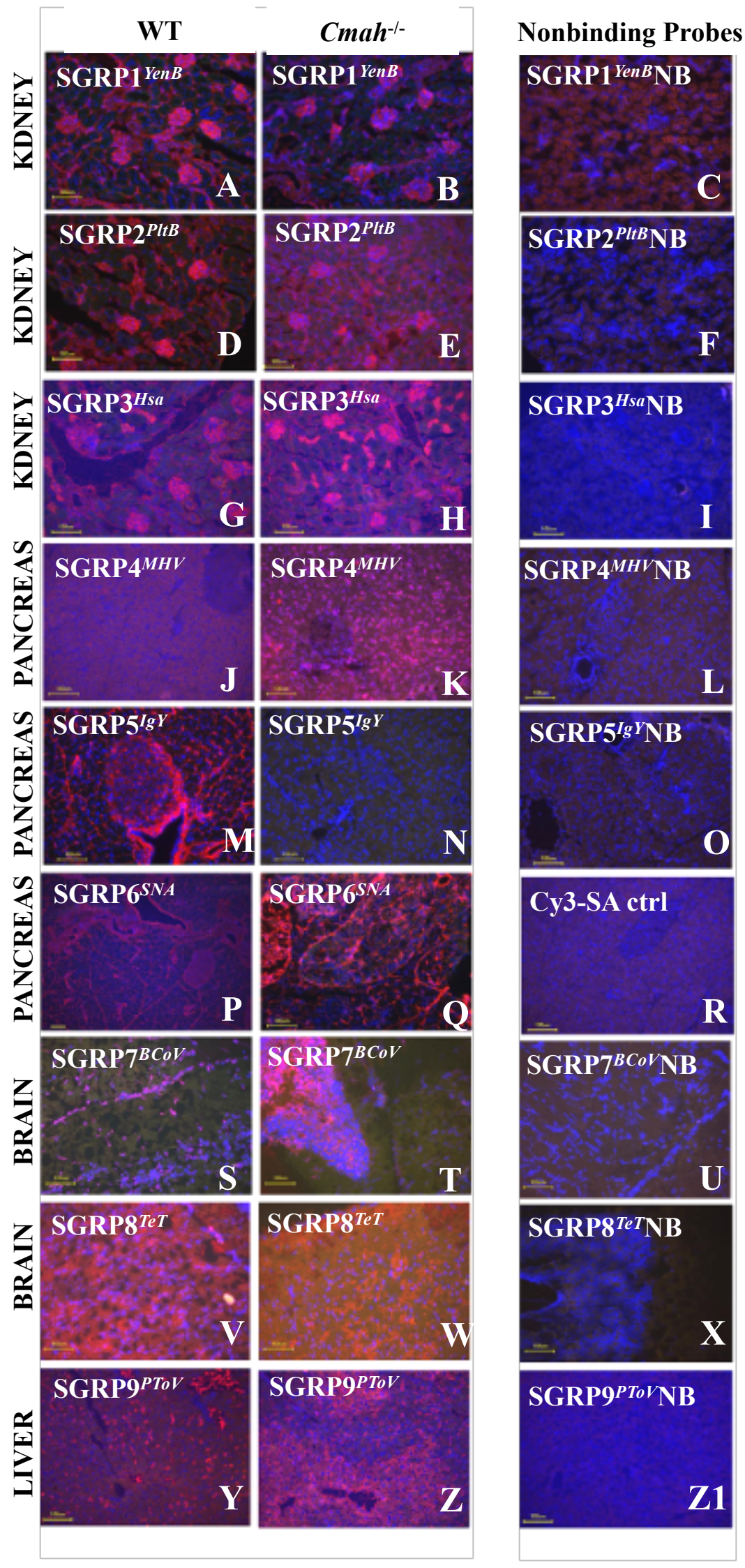

Figure 4 
bioRxiv preprint doi: https://doi.org/10.1101/2021.05.28.446202; this version posted May 28, 2021. The copyright holder for this preprint (which was not certified by peer review) is the author/funder, who has granted bioRxiv a license to display the preprint in perpetuity. It is made available under aCC-BY-NC-ND 4.0 International license.

Guinea

CMAH'--

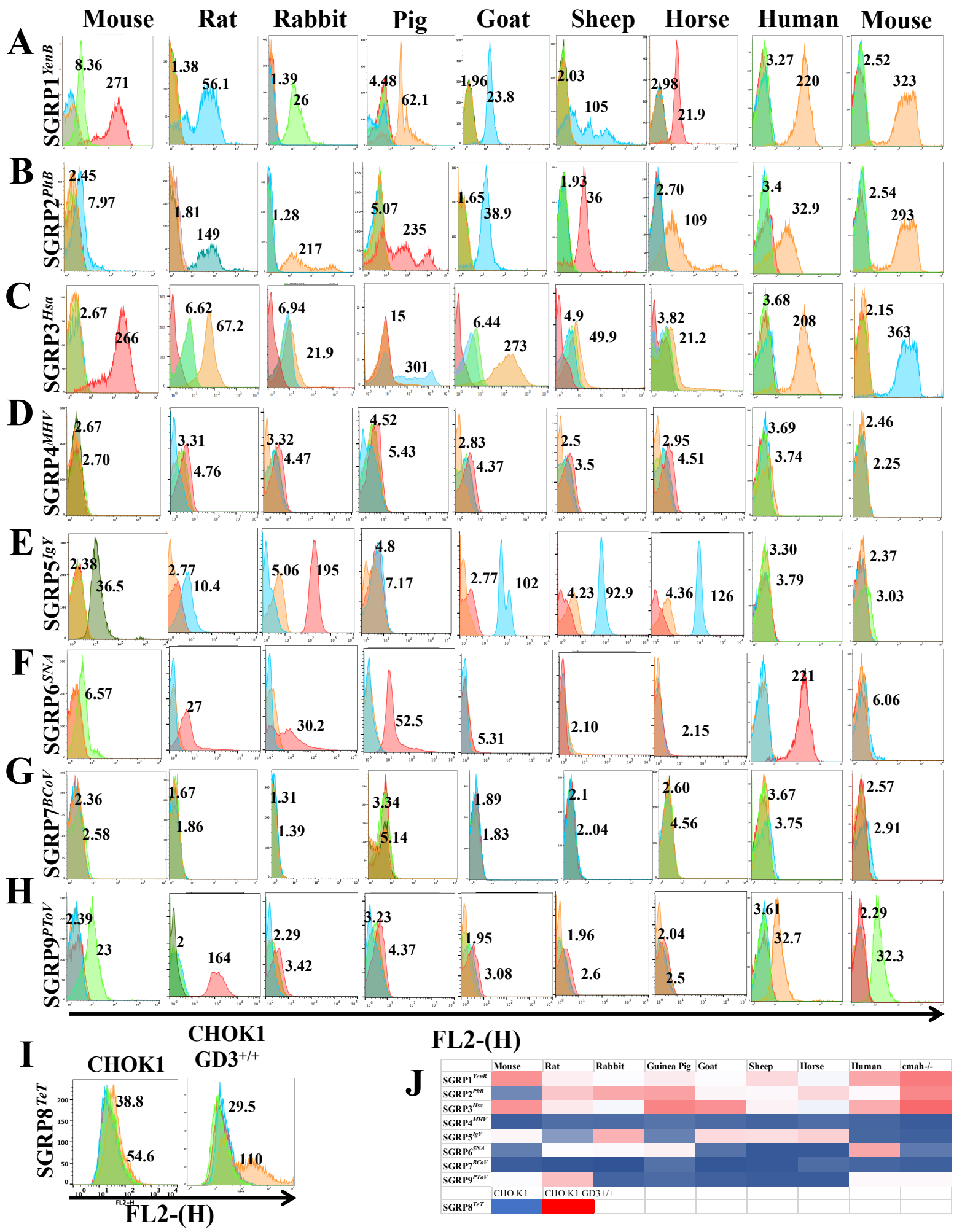

Figure 5 